\title{
Shallow-water sloshing in a moving vessel with variable cross-section and wetting-drying using an extension of George's well-balanced finite volume solver
}

\author{
Hamid Alemi Ardakani, ${ }^{1}$ Thomas J. Bridges \& Matthew R. Turner
}

Department of Mathematics, University of Surrey, Guildford, Surrey GU2 7XH, UK

\begin{abstract}
A class of augmented approximate Riemann solvers due to George (2008) (J. Comput. Phys. 227, 3089-3113) is extended to solve the shallow-water equations in a moving vessel with variable bottom topography and variable cross-section with wetting and drying. A class of Roe-type upwind solvers for the system of balance laws is derived which respects the steady-state solutions. The numerical solutions of the new adapted augmented f-wave solvers are validated against the Roe-type solvers. The theory is extended to solve the shallow-water flows in moving vessels with arbitrary cross-section with influx-efflux boundary conditions motivated by the shallow-water sloshing in the ocean wave energy converter (WEC) proposed by Offshore Wave Energy Ltd (OWEL) [1]. A fractional step approach is used to handle the time-dependent forcing functions. The numerical solutions are compared to an extended new Roe-type solver for the system of balance laws with a time-dependent source function. The shallow-water sloshing finite volume solver can be coupled to a Runge-Kutta integrator for the vessel motion.
\end{abstract}

Keywords: Shallow-water sloshing; Hyperbolic conservation laws; Finite-volume method; F-wave-propagation; Wetting and drying; Roe solver.

\section{Introduction}

A class of high resolution wave-propagation finite volume methods is developed in [20] for multidimensional hyperbolic systems. These methods are based on solving Riemann problems for waves that define both first order updates to cell averages and also second order corrections which can be modified by limiter functions to obtain high resolution numerical solutions. The wave-propagation algorithms are modified in [5] for conservation laws and balance laws with spatially varying flux functions and are called f-wave-propagation methods. The main novel feature of the modified algorithms is to solve the Riemann problems by decomposition of the jump in the flux functions into waves propagating out from each grid cell interface instead of

\footnotetext{
${ }^{1}$ Corresponding author. Email address: h.alemiardakani@surrey.ac.uk (H. Alemi Ardakani).
} 
decomposition of the jump in cell averages. In [11, 12] a class of augmented approximate Riemann solvers is developed for the single layer shallow water equations in the presence of a variable bottom surface using the f-wave-propagation algorithm. The solver is based on a decomposition of an augmented solution vector including the depth, momentum, momentum flux and the bottom surface. This solver is well-balanced, maintains depth non-negativity and extends to Riemann problems with an initial dry state.

The shallow-water equations over variable bottom topography and cross-section form a set of nonlinear hyperbolic conservation laws with geometric source terms due to the arbitrary crosssection constraining the flow. For a vessel with a symmetric rectangular cross-section with regard to a vertical plane passing through its longitudinal horizontal axis, the equations take the form

$$
\begin{aligned}
(h \sigma)_{t}+(h u \sigma)_{x} & =0 \\
(h u \sigma)_{t}+\left(h u^{2} \sigma+\frac{1}{2} g h^{2} \sigma\right)_{x} & =\frac{1}{2} g h^{2} \sigma_{x}-g h \sigma \beta_{x},
\end{aligned}
$$

where $g$ is the gravitational constant, $h(x, t)$ is the fluid depth, $u(x, t)$ is the vertically averaged horizontal fluid velocity, $\beta(x)$ is the bottom topography, and $\sigma(x)$ is the breadth function such that the vessel walls are defined by the equations $y= \pm \frac{1}{2} \sigma(x)$. Here the subscripts denote partial derivatives. A schematic of the configuration of interest is shown in Figure 1. The shallow-water equations (1.1) belong to the hyperbolic systems of the form

$$
\mathrm{q}_{t}+f(\mathbf{q}, x)_{x}=\Psi(\mathbf{q}, x)
$$

where $\mathrm{q} \in \mathbb{R}^{m}$ is a vector of conserved quantities, $f(\mathrm{q}, x) \in \mathbb{R}^{m}$ is the vector of corresponding fluxes, and $\Psi(\mathrm{q}, x) \in \mathbb{R}^{m}$ is a vector of source terms. Non-trivial steady state solutions to (1.2) exist due to a balance of the flux gradient and the momentum source term due to variable topography and cross-section. Preserving the non-trivial steady states, or resolving small perturbations to them in the numerical solver when

$$
f(\mathrm{q}, x)_{x} \approx \Psi(\mathrm{q}, x)
$$

and both terms are relatively large is a well-known difficulty which has received considerable studies, for instance see $[30,10,5,6,22,23,12]$. The other difficulty which arises in the numerical solution of the balance laws like (1.1) is the appearance and movement of a wet/dry front with vanishing or zero depth regions ahead or behind of the front [12]. Preserving depth non-negativity while maintaining mass conservation is particularly difficult with most standard Riemann solvers [12]. In [12] an augmented finite volume Riemann solver is developed for the shallow water equations over variable topography which preserves the steady states and maintains depth positivity in the Riemann solution along with satisfying other standard properties sought in Riemann solvers such as accurate capturing of wet/dry fronts and entropy requirements in the presence of large rarefaction waves.

This paper starts with the derivation of the shallow-water equations over a variable bottom topography and variable cross-section in moving coordinates with horizontal acceleration in $\S 2$. The interest in this paper is to develop an adapted version of the augmented f-wave finite volume solver of [12] for the shallow water equations (1.1) over variable cross-section and variable bottom topography which could handle Riemann problems with an initial dry state, and then modify the new solver to include a time-dependent source function to simulate shallow water sloshing with prescribed or coupled surge motion. The new augmented f-wave solver is 


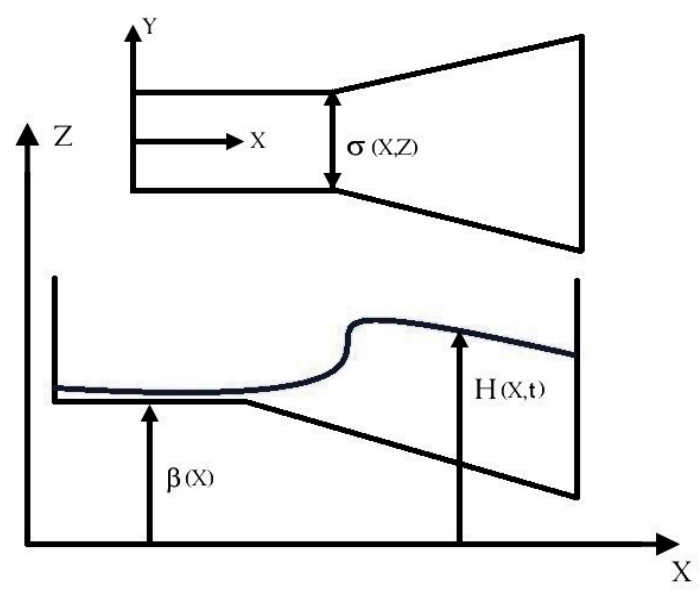

Figure 1: Schematic of one-layer shallow-water sloshing over variable topography and crosssection in a stationary vessel.

validated against the Roe-type solver of [17] which is reviewed in $\S 3$. In $\S 4$ a new version of the Roe-type solver of [17] is derived for the shallow-water equations (1.1) with a time-dependent surge forcing function in the momentum equation (see equation (2.10)). The time-dependent augmented f-wave solver is then validated against the new Roe-type solver of $\S 4$. A review of the $f$-wave finite volume methods of $[5,22]$ is given in $\S 5$. The detailed derivation of the new augmented f-wave finite volume solver for the shallow-water equations (1.1) is presented in §6. Section 7 is devoted to the numerical simulations and validations of the discussed solvers. Finally, some concluding remarks are given in $\S 8$.

\section{Governing equations}

The derivation of the governing equations for two-layer shallow-water flows through channels with irregular geometry is given in [7] by defining a control volume of fluid and applying the mass and the momentum conservation laws to each fluid layer. A similar argument can be used to derive the system of balance laws for one-layer shallow-water sloshing equations in a moving vessel with an arbitrary cross-section. The following notation is used. There are two reference coordinates: the spatial (inertial) frame has coordinates $\mathbf{X}=(X, Y, Z)$ and the body frame has coordinates $\mathbf{x}=(x, y, z)$. The whole system has a uniform translation, denoted $\xi(t)$, in the $x$-direction; hence

$$
X=x+\xi, \quad Y=y \quad \text { and } \quad Z=z .
$$

Variables $\beta(x)$ and $\sigma(x, z)$ are, respectively, bottom and breadth functions. i.e., the vessel bottom is defined by the surface of equation $z=\beta(x)$ and vessel walls by the equations $y=$ $\pm \frac{1}{2} \sigma(x, z)$. The fluid density is denoted by $\rho$ and is assumed to be a constant. The variables $\mathcal{A}(x, t)$ and $H(x, t)=\beta(x)+h(x, t)$ represent the wetted cross-section of the vessel and the wave elevation with respect to $z=0$, at the section of coordinate $x$ at time $t$. Therefore $\mathcal{A}(x, t)$ 
and $h(x, t)$ are related through the equation

$$
\mathcal{A}(x, t)=\int_{\beta(x)}^{H(x, t)} \sigma(x, z) d z .
$$

The volume flux or discharge is represented by $\mathcal{Q}(x, t)$ through the equation

$$
\mathcal{Q}(x, t)=\int_{\beta(x)}^{H(x, t)} u(x, t) \sigma(x, z) d z=u(x, t) \mathcal{A}(x, t) .
$$

Consider a control volume of fluid $\mathcal{R}$ which is delimited at time $t$ by two arbitrary cross-sections of coordinates $x=x_{1}$ and $x=x_{2}\left(x_{1}<x_{2}\right)$. The portion of the boundary of $\mathcal{R}$ corresponding to the cross-sections at $x=x_{1}$ and $x=x_{2}$ is denoted by $\Gamma_{1}$ and $\Gamma_{2}$, respectively, and $\Gamma_{w}$ the boundaries defined by the walls. Also $\Gamma_{s}$ and $\Gamma_{\beta}$ denote, respectively, the portion of the free surface and the bottom surface between sections $x=x_{1}$ and $x=x_{2}$.

The conservation of mass can be expressed under integral form as follows

$$
\frac{\partial}{\partial t} \int_{\mathcal{R}} \rho d \mathcal{R}+\int_{\partial \mathcal{R}} \rho u(x, t) n_{1} d A=0,
$$

where $n_{1}$ represents the first component of the outward normal unit vector. This equation can be converted to the following form

$$
\int_{x_{1}}^{x_{2}} \frac{\partial}{\partial t}(\rho \mathcal{A}) d x-\rho \mathcal{Q}\left(x_{1}, t\right)+\rho \mathcal{Q}\left(x_{2}, t\right)=\int_{x_{1}}^{x_{2}}\left(\frac{\partial}{\partial t}(\rho \mathcal{A})+\frac{\partial}{\partial x}(\rho \mathcal{Q})\right) d x=0 .
$$

And hence

$$
\frac{\partial \mathcal{A}}{\partial t}+\frac{\partial \mathcal{Q}}{\partial x}=0
$$

The momentum conservation law can also be expressed in integral form as follows

$$
\frac{\partial}{\partial t} \int_{\mathcal{R}} u(x, t) \rho d \mathcal{R}+\int_{\partial \mathcal{R}} \rho u(x, t)^{2} n_{1} d A=\int_{\partial \mathcal{R}}-p(x, z, t) n_{1} d A-\int_{\mathcal{R}} \rho \ddot{\xi} d \mathcal{R}
$$

where $\ddot{\xi}$ represents the horizontal acceleration of the vessel relative to the spatial frame, and $p(x, z)$ is the pressure of the fluid layer, which is assumed to be hydrostatic, taking the form

$$
p(x, z, t)=\rho g(H(x, t)-z) .
$$

The first integral in (2.8) can be converted to the following form

$$
\frac{\partial}{\partial t} \int_{\mathcal{R}} u(x, t) \rho d \mathcal{R}=\frac{\partial}{\partial t} \int_{x_{1}}^{x_{2}} \int_{\beta(x)}^{H(x, t)} \rho u(x, t) \sigma(x, z) d z d x=\frac{\partial}{\partial t} \int_{x_{1}}^{x_{2}} \rho \mathcal{Q}(x, t) d x .
$$

The second integral in (2.8) reads

$$
\int_{\partial \mathcal{R}} \rho u(x, t)^{2} n_{1} d A=-\rho u\left(x_{1}, t\right) \mathcal{Q}\left(x_{1}, t\right)+\rho u\left(x_{2}, t\right) \mathcal{Q}\left(x_{2}, t\right)=\int_{x_{1}}^{x_{2}} \rho \frac{\partial}{\partial x}\left(\frac{\mathcal{Q}^{2}}{\mathcal{A}}\right) d x
$$


And the pressure integral in (2.8) can be converted to the following form

$$
\int_{\partial \mathcal{R}}-p(x, z, t) n_{1} d A=-\int_{\Gamma_{1}} p n_{1} d \Gamma_{1}-\int_{\Gamma_{2}} p n_{1} d \Gamma_{2}-\int_{\Gamma_{w}} p n_{1} d \Gamma_{w}-\int_{\Gamma_{\beta}} p n_{1} d \Gamma_{\beta}-\int_{\Gamma_{s}} p n_{1} d \Gamma_{s},
$$

where

$$
-\int_{\Gamma_{1}} p n_{1} d \Gamma_{1}=\rho g \widetilde{\Gamma}_{1}\left(x_{1}, t\right), \quad \widetilde{\Gamma}_{1}(x, t)=\int_{\beta(x)}^{H(x, t)}(H(x, t)-z) \sigma(x, z) d z,
$$

and

$$
\begin{aligned}
-\int_{\Gamma_{2}} p n_{1} d \Gamma_{2} & =-\rho g \widetilde{\Gamma}_{1}\left(x_{2}, t\right), \\
-\int_{\Gamma_{w}} p n_{1} d \Gamma_{w} & =\int_{x_{1}}^{x_{2}} \rho g \widetilde{\Gamma}_{w}(x, t) d x, \quad \widetilde{\Gamma}_{w}(x, t)=\int_{\beta(x)}^{H(x, t)}(H(x, t)-z) \frac{\partial \sigma(x, z)}{\partial x} d z, \\
-\int_{\Gamma_{s}} p n_{1} d \Gamma_{s} & =0, \\
-\int_{\Gamma_{\beta}} p n_{1} d \Gamma_{\beta} & =-\int_{x_{1}}^{x_{2}} \rho g(H(x, t)-\beta(x)) \sigma(x, z) \frac{d \beta}{d x} d x .
\end{aligned}
$$

Also the last integral in (2.8) reads

$$
-\int_{\mathcal{R}} \rho \ddot{\xi} d \mathcal{R}=-\int_{x_{1}}^{x_{2}} \rho \ddot{\xi} \mathcal{A}(x, t) d x
$$

Now the momentum conservation law (2.8) can be written in differential form as

$$
\frac{\partial \mathcal{Q}}{\partial t}+\frac{\partial}{\partial x}\left(\frac{\mathcal{Q}^{2}}{\mathcal{A}}\right)=-g \frac{\partial \widetilde{\Gamma}_{1}}{\partial x}+g \widetilde{\Gamma}_{w}-g h \sigma \beta_{x}-\ddot{\xi} \mathcal{A}
$$

\subsection{Governing equations with rectangular cross-section}

For a vessel with rectangular cross-section, $\sigma=\sigma(x)$, we have the following simplifications:

$$
\begin{aligned}
\mathcal{A}(x, t) & =h(x, t) \sigma(x), \\
\mathcal{Q}(x, t) & =h(x, t) u(x, t) \sigma(x)=\mathcal{A}(x, t) u(x, t), \\
\widetilde{\Gamma}_{1}(x, t) & =\frac{1}{2} \sigma(x) h(x, t)^{2}, \\
\widetilde{\Gamma}_{w}(x, t) & =\frac{1}{2} h(x, t)^{2} \sigma(x) .
\end{aligned}
$$

Substitution of these expressions into the shallow-water equations (2.7) and (2.9) gives

$$
\begin{aligned}
(h \sigma)_{t}+(h u \sigma)_{x} & =0 \\
(h u \sigma)_{t}+\left(h u^{2} \sigma+\frac{1}{2} g h^{2} \sigma\right)_{x} & =\frac{1}{2} g h^{2} \sigma_{x}-g h \sigma \beta_{x}-\ddot{\xi} h \sigma .
\end{aligned}
$$

For a stationary vessel $\ddot{\xi}=0$ and the shallow-water equations (2.10) recover the balance law given in (1.1). 


\section{Review of the derivation of the Roe-type solver of Hernandez- Duenas \& Karni}

In $[13,10]$ approximate Riemann solutions of the shallow water equations over constant and variable cross-section based on the Roe linearization method are presented. In this section we follow the approach of [17] to derive a Roe-type upwind scheme for the hyperbolic system (1.1) with upwinding of the geometric source terms $[26,27]$. The derivation for the more general case where $\sigma=\sigma(x, z)$ is given in [17] but here we review the derivation for the case $\sigma=\sigma(x)$.

The conservative and quasilinear forms of the hyperbolic system (1.1) at the differential level can be related as

$$
\left[\begin{array}{c}
h u \sigma \\
h u^{2} \sigma+\frac{1}{2} g h^{2} \sigma
\end{array}\right]_{x}=\left(\begin{array}{cc}
0 & 1 \\
-u^{2}+g h & 2 u
\end{array}\right)\left[\begin{array}{c}
h \sigma \\
h u \sigma
\end{array}\right]_{x}+\left[\begin{array}{c}
0 \\
-\frac{1}{2} g h^{2} \sigma_{x}
\end{array}\right] .
$$

To obtain a discrete analogue of (3.11) the discrete version of the Fundamental Theorem of Calculus (FTC) which is

$$
\begin{aligned}
\Delta \int_{a(x)}^{b(x)} f(x, z) d z \equiv & \int_{a_{r}}^{b_{r}} f\left(x_{r}, z\right) d z-\int_{a_{l}}^{b_{l}} f\left(x_{l}, z\right) d z=\frac{1}{2}\left(\int_{a_{l}}^{b_{l}}+\int_{a_{r}}^{b_{r}}\right) \Delta f(z) d z \\
& +\int_{b_{l}}^{b_{r}} \bar{f}(z) d z-\int_{a_{l}}^{a_{r}} \bar{f}(z) d z
\end{aligned}
$$

is used [17]. We have used here and in what follows $\Delta()=()_{r}-()_{l}$, and $\left(^{-}\right)=$ $\frac{1}{2}\left(()_{r}+()_{l}\right)$.

The discrete version of (3.11) requires the flux difference $\Delta\left(h u^{2} \sigma+\frac{1}{2} g h^{2} \sigma\right)$. We begin with the term $h u^{2} \sigma$ and seek a linearization of the form

$$
\Delta\left(h u^{2} \sigma\right)=-\widehat{u}^{2} \Delta(h \sigma)+2 \widehat{u} \Delta(h u \sigma),
$$

that is satisfied by

$$
\widehat{u}=\frac{\sqrt{h_{l} \sigma_{l}} u_{l}+\sqrt{h_{r} \sigma_{r}} u_{r}}{\sqrt{h_{l} \sigma_{l}}+\sqrt{h_{r} \sigma_{r}}} .
$$

Next, the discrete FTC (3.12) is applied to express $\Delta\left(\frac{1}{2} g h^{2} \sigma\right)$. We have

$$
\begin{aligned}
\Delta\left(\frac{1}{2} g h^{2} \sigma\right)= & g \Delta \widetilde{\Gamma}_{1}=\Delta \int_{\beta}^{H} g(H-z) \sigma(x) d z, \\
& =\frac{g}{2}\left(\int_{\beta_{l}}^{H_{l}}+\int_{\beta_{r}}^{H_{r}}\right) \Delta((H-z) \sigma(x)) d z+g \int_{H_{l}}^{H_{r}} \overline{(H-z) \sigma(x)} d z \\
& -g \int_{\beta_{l}}^{\beta_{r}} \overline{(H-z) \sigma(x)} d z, \\
& =\frac{g}{2}\left(\int_{\beta_{l}}^{H_{l}}+\int_{\beta_{r}}^{H_{r}}\right)(\bar{\sigma}(x) \Delta H+(\bar{H}-z) \Delta \sigma(x)) d z+g \int_{H_{l}}^{H_{r}} \overline{(H-z) \sigma(x)} d z \\
& -g \int_{\beta_{l}}^{\beta_{r}} \overline{(H-z) \sigma(x)} d z, \\
& =g \widehat{\mathcal{A}} \Delta H+\Delta x \widehat{\Gamma}_{w}+\widehat{G}-g \widehat{h \sigma} \Delta \beta,
\end{aligned}
$$


where the identity $\Delta(\nu \xi)=\bar{\nu} \Delta \xi+\bar{\xi} \Delta \nu$ is used in the third line of (3.14) and

$$
\begin{aligned}
& \widehat{\mathcal{A}}=\frac{1}{2}\left(\int_{\beta_{l}}^{H_{l}}+\int_{\beta_{r}}^{H_{r}}\right) \bar{\sigma}(x) d z, \quad \Delta x \widehat{\Gamma}_{w}=\frac{g}{2}\left(\int_{\beta_{l}}^{H_{l}}+\int_{\beta_{r}}^{H_{r}}\right)(\bar{H}-z) \Delta \sigma(x) d z, \\
& \widehat{G}=g \int_{H_{l}}^{H_{r}} \overline{(H-z) \sigma(x)} d z, \quad g \widehat{h \sigma} \Delta \beta=g \int_{\beta_{l}}^{\beta_{r}} \overline{(H-z) \sigma(x)} d z .
\end{aligned}
$$

In order to express $\Delta H$ in (3.14) in terms of the conserved variables, the discrete FTC is applied to $\mathcal{A}(x, t)$ resulting in

$$
\Delta \mathcal{A}=\frac{1}{2}\left(\int_{\beta_{l}}^{H_{l}}+\int_{\beta_{r}}^{H_{r}}\right) \Delta \sigma(x) d z+\int_{H_{l}}^{H_{r}} \bar{\sigma}(x) d z-\int_{\beta_{l}}^{\beta_{r}} \bar{\sigma}(x) d z=\Delta x \widehat{h I}+\widehat{\sigma} \Delta(h+\beta)-\widehat{\sigma} \Delta \beta,
$$

where

$$
\Delta x \widehat{h I}=\frac{1}{2}\left(\int_{\beta_{l}}^{H_{l}}+\int_{\beta_{r}}^{H_{r}}\right) \Delta \sigma(x) d z, \quad \widehat{\sigma} \Delta(h+\beta)=\int_{H_{l}}^{H_{r}} \bar{\sigma}(x) d z, \quad \widehat{\sigma} \Delta \beta=\int_{\beta_{l}}^{\beta_{r}} \bar{\sigma}(x) d z,
$$

and hence

$$
\Delta H=\frac{1}{\widehat{\sigma}}(\Delta \mathcal{A}+\widehat{\sigma} \Delta \beta-\Delta x \widehat{h I})
$$

and $\Delta\left(g \widetilde{\Gamma}_{1}\right)$ becomes

$$
\Delta\left(g \widetilde{\Gamma}_{1}\right)=\widehat{c}^{2} \Delta \mathcal{A}+\widehat{c}^{2}(\widehat{\sigma} \Delta \beta-\Delta x \widehat{h I})+\widehat{G}+\Delta x \widehat{\Gamma}_{w}-g \widehat{h \sigma} \Delta \beta,
$$

where $\widehat{c}^{2}=\frac{g \widehat{\mathcal{A}}}{\widehat{\sigma}}$. Now the discrete version of (3.11) reads

$$
\Delta\left[\begin{array}{c}
h u \sigma \\
h u^{2} \sigma+\frac{1}{2} g h^{2} \sigma
\end{array}\right]=\left(\begin{array}{cc}
0 & 1 \\
-\widehat{u}^{2}+\widehat{c}^{2} & 2 \widehat{u}
\end{array}\right)\left[\begin{array}{c}
\Delta(h \sigma) \\
\Delta(h u \sigma)
\end{array}\right]+\left[\begin{array}{c}
0 \\
\widehat{c}^{2}(\widehat{\sigma} \Delta \beta-\Delta x \widehat{h I})+\widehat{G}+\Delta x \widehat{\Gamma}_{w}-g \widehat{h \sigma} \Delta \beta
\end{array}\right] .
$$

To develop a well-balanced scheme the following relation should be satisfied

$$
\Delta\left[\begin{array}{c}
h u \sigma \\
h u^{2} \sigma+\frac{1}{2} g h^{2} \sigma
\end{array}\right]-\left[\begin{array}{c}
0 \\
\Delta x \widehat{\Gamma}_{w}-g \widehat{h \sigma} \Delta \beta
\end{array}\right]=\sum_{k}\left(\widehat{\lambda}^{k} \widehat{\alpha}_{k}-\widehat{\beta}_{k}\right) \widehat{r}^{k}
$$

where $\widehat{r}^{1}=\left[\begin{array}{ll}1 & \widehat{\lambda}^{1}\end{array}\right]^{T}, \widehat{r}^{2}=\left[\begin{array}{ll}1 & \widehat{\lambda}^{2}\end{array}\right]^{T}$, and $\widehat{\alpha}_{k}$ can be obtained from the usual condition

$$
\Delta\left[\begin{array}{c}
h \sigma \\
h u \sigma
\end{array}\right]=\left(\begin{array}{cc}
1 & 1 \\
\widehat{\lambda}^{1} & \widehat{\lambda}^{2}
\end{array}\right)\left[\begin{array}{c}
\widehat{\alpha}_{1} \\
\widehat{\alpha}_{2}
\end{array}\right] \text { with } \quad \widehat{\lambda}^{1}=\widehat{u}-\widehat{c}, \quad \widehat{\lambda}^{2}=\widehat{u}+\widehat{c},
$$

and so

$$
\widehat{\alpha}_{1}=\frac{(\widehat{u}+\widehat{c}) \Delta(h \sigma)-\Delta(h u \sigma)}{2 \widehat{c}}, \quad \widehat{\alpha}_{2}=\frac{-(\widehat{u}-\widehat{c}) \Delta(h \sigma)+\Delta(h u \sigma)}{2 \widehat{c}} .
$$

Now substitution of (3.18) and (3.20) into (3.19) gives

$$
\widehat{\beta}_{1}=\frac{1}{2 \widehat{c}}\left(\widehat{c}^{2}(\widehat{\sigma} \Delta \beta-\Delta x \widehat{h I})+\widehat{G}\right), \quad \widehat{\beta}_{2}=-\frac{1}{2 \widehat{c}}\left(\widehat{c}^{2}(\widehat{\sigma} \Delta \beta-\Delta x \widehat{h I})+\widehat{G}\right)
$$


Finally the expressions used so far in the derivation for $\sigma=\sigma(x)$ reduces to

$$
\widehat{\mathcal{A}}=\bar{h} \bar{\sigma}, \quad \widehat{\sigma}=\bar{\sigma}, \quad \widehat{c}^{2}=g \bar{h}, \quad \widehat{G}=\frac{1}{4} g \Delta \sigma(\Delta(\beta+h))^{2}, \quad \Delta x \widehat{h I}=\Delta \sigma \bar{h} .
$$

Now the total fluctuation in the first wave family for the steady state at rest, $u=0, \Delta H=0$, becomes

$$
\widehat{\alpha}_{1} \widehat{\lambda}^{1}-\widehat{\beta}_{1}=-\frac{1}{2} \widehat{c}(\bar{\sigma} \Delta h+\widehat{\sigma} \Delta \beta)+\frac{1}{2} \Delta(h u \sigma),
$$

and the total fluctuation in the second wave family becomes

$$
\widehat{\alpha}_{2} \widehat{\lambda}^{2}-\widehat{\beta}_{2}=\frac{1}{2} \widehat{c}(\bar{\sigma} \Delta h+\widehat{\sigma} \Delta \beta)+\frac{1}{2} \Delta(h u \sigma),
$$

and so the first of (3.19) is recovered, which insures that the steady state solutions at rest are recognized.

The shallow-water equations (1.1) can be written as

$$
\mathrm{q}_{t}+\mathbb{A}(\mathrm{q}) \mathrm{q}_{x}=\Psi(\mathrm{q}, x)
$$

where

$$
\mathbf{q}=\left[\begin{array}{c}
h \sigma \\
h u \sigma
\end{array}\right], \quad \mathbb{A}(\mathbf{q})=\left[\begin{array}{cc}
0 & 1 \\
-u^{2}+g h & 2 u
\end{array}\right], \quad \Psi(\mathbf{q}, x)=\left[\begin{array}{c}
0 \\
g h^{2} \sigma_{x}-g h \sigma \beta_{x}
\end{array}\right] .
$$

Finally the numerical scheme takes the general form

$$
\mathbb{Q}_{i}^{n+1}=\mathbb{Q}_{i}^{n}-\frac{\Delta t}{\Delta x}\left(\mathbb{A}^{+} \Delta \mathbb{Q}_{i-1 / 2}+\mathbb{A}^{-} \Delta \mathbb{Q}_{i+1 / 2}\right)
$$

where

$$
\begin{aligned}
\mathbb{Q}_{i}^{n} & =\frac{1}{\Delta x} \int_{\mathcal{C}_{i}} \mathrm{q}\left(x, t^{n}\right) d x, \quad \text { with } \quad \mathcal{C}_{i}=\left[x_{i-1 / 2}, x_{i+1 / 2}\right], \quad \Delta x=\left(x_{i+1 / 2}-x_{i-1 / 2}\right), \\
\Delta t & =\left(t^{n+1}-t^{n}\right),
\end{aligned}
$$

and the domain is partitioned into grid cells $\mathcal{C}$, and

$$
\mathbb{A}^{+} \Delta \mathbb{Q}_{i-1 / 2}=\sum_{\widehat{\lambda}^{k}>0}\left[\left(\widehat{\alpha}_{k} \widehat{\lambda}^{k}-\widehat{\beta}_{k}\right) \widehat{r}^{k}\right]_{i-1 / 2}, \quad \mathbb{A}^{-} \Delta \mathbb{Q}_{i-1 / 2}=\sum_{\widehat{\lambda}^{k}<0}\left[\left(\widehat{\alpha}_{k} \widehat{\lambda}^{k}-\widehat{\beta}_{k}\right) \widehat{r}^{k}\right]_{i-1 / 2} .
$$

It is known that the linearized Riemann solvers like Roe-type solvers consist only of discontinuities, with no rarefaction waves. So this may lead to a violation of the entropy condition. The Roe-type Riemann solver presented in this section can be implemented with Harten-Hyman [15] entropy fix or local Lax-Friedrichs (LFF) method as is discussed in [22].

A fractional step approach can be used to solve the hyperbolic shallow water system (2.10) with a time-dependent source function. After each time step of the hyperbolic problem a time step is taken in which the momentum equation is adjusted due to the horizontal acceleration of the vessel. For given $\mathbb{Q}_{i}^{n}$ apply the Roe-type scheme (3.24) to update $\mathbb{Q}_{i}$ over time $\Delta t$ and call the new vector $\mathbb{Q}_{i}^{\star}$. Then update $\mathbb{Q}_{i}^{\star}$ to $\mathbb{Q}_{i}^{n+1}$ by solving

$$
\left.\begin{array}{rl}
\frac{\partial}{\partial t}(h \sigma) & =0, \\
\frac{\partial}{\partial t}(h u \sigma) & =-\ddot{\xi} h \sigma .
\end{array}\right\}
$$




\section{A new Roe-type solver with a time-dependent source func- tion}

Instead of using the proposed fractional step approach of $\S 3$ in order to handle the timedependent source functions, the forcing function can be incorporated into the Roe solver. In this section the derivation of a new Roe-type solver for the shallow-water equations over variable topography and variable cross-section under the surge forcing function is given.

The starting point is the shallow-water equations (2.10) which can be written as

$$
\begin{aligned}
(h \sigma)_{t}+(h \sigma(u+\dot{\xi}))_{x} & =\dot{\xi}(h \sigma)_{x}, \\
(h \sigma(u+\dot{\xi}))_{t}+\left(h u^{2} \sigma+\frac{1}{2} g h^{2} \sigma+\dot{\xi} h u \sigma\right)_{x} & =\frac{1}{2} g h^{2} \sigma_{x}-g h \sigma \beta_{x} .
\end{aligned}
$$

Then the conservative and quasilinear forms of the hyperbolic system (4.27) at the differential level can be related as

$$
\left[\begin{array}{c}
h \sigma(u+\dot{\xi}) \\
h u^{2} \sigma+\frac{1}{2} g h^{2} \sigma+\dot{\xi} h u \sigma
\end{array}\right]_{x}=\left(\begin{array}{cc}
0 & 1 \\
-(u+\dot{\xi})^{2}+g h & 2 u+\dot{\xi}
\end{array}\right)\left[\begin{array}{c}
h \sigma \\
h \sigma(u+\dot{\xi})
\end{array}\right]_{x}+\left[\begin{array}{c}
0 \\
-\frac{1}{2} g h^{2} \sigma_{x}
\end{array}\right] .
$$

The discrete version of (4.28) requires the flux difference $\Delta\left(h u^{2} \sigma+\frac{1}{2} g h^{2} \sigma+\dot{\xi} h u \sigma\right)$. We begin with the term $h u \sigma(u+\dot{\xi})$ and seek a linearization of the form

$$
\Delta(h u \sigma(u+\dot{\xi}))=-(\widehat{u}+\dot{\xi})^{2} \Delta(h \sigma)+(2 \widehat{u}+\dot{\xi}) \Delta(h \sigma(u+\dot{\xi}))
$$

which is satisfied by (3.13). Also $\Delta\left(\frac{1}{2} g h^{2} \sigma\right)$ takes the form (3.17). And hence the discrete version of (4.28) reads

$$
\begin{aligned}
\Delta\left[\begin{array}{c}
h \sigma(u+\dot{\xi}) \\
h u^{2} \sigma+\frac{1}{2} g h^{2} \sigma+\dot{\xi} h u \sigma
\end{array}\right]= & \left(\begin{array}{cc}
0 & 1 \\
-(\widehat{u}+\dot{\xi})^{2}+\widehat{c}^{2} & 2 \widehat{u}+\dot{\xi}
\end{array}\right)\left[\begin{array}{c}
\Delta(h \sigma) \\
\Delta(h \sigma(u+\dot{\xi}))
\end{array}\right] \\
& +\left[\begin{array}{c}
0 \\
\widehat{c}^{2}(\widehat{\sigma} \Delta \beta-\Delta x \widehat{h I})+\widehat{G}+\Delta x \widehat{\Gamma}_{w}-g \widehat{h \sigma} \Delta \beta
\end{array}\right] .
\end{aligned}
$$

To derive a well-balanced scheme the following relation should be satisfied

$$
\Delta\left[\begin{array}{c}
h \sigma(u+\dot{\xi}) \\
h u^{2} \sigma+\frac{1}{2} g h^{2} \sigma+\dot{\xi} h u \sigma
\end{array}\right]-\left[\begin{array}{c}
\Delta(\dot{\xi} h \sigma) \\
\Delta x \widehat{\Gamma}_{w}-g h \sigma \Delta \beta
\end{array}\right]=\sum_{k}\left(\widehat{\lambda}^{k} \widehat{\alpha}_{k}-\widehat{\beta}_{k}\right) \widehat{r}^{k},
$$

where $\widehat{r}^{1}=\left[\begin{array}{ll}1 & \widehat{\lambda}^{1}\end{array}\right]^{T}, \widehat{r}^{2}=\left[\begin{array}{ll}1 & \widehat{\lambda}^{2}\end{array}\right]^{T}$, and $\widehat{\alpha}_{k}$ can be obtained from

$$
\Delta\left[\begin{array}{c}
h \sigma \\
h \sigma(u+\dot{\xi})
\end{array}\right]=\left(\begin{array}{cc}
1 & 1 \\
\hat{\lambda}^{1} & \widehat{\lambda}^{2}
\end{array}\right)\left[\begin{array}{l}
\widehat{\alpha}_{1} \\
\widehat{\alpha}_{2}
\end{array}\right] \text { with }\left\{\begin{array}{l}
\widehat{\lambda}^{1}=\widehat{u}+\frac{1}{2} \dot{\xi}-\frac{1}{2} \sqrt{-3 \dot{\xi}^{2}-4 \widehat{u} \dot{\xi}+4 g \bar{h}} \\
\hat{\lambda}^{2}=\widehat{u}+\frac{1}{2} \dot{\xi}+\frac{1}{2} \sqrt{-3 \dot{\xi}^{2}-4 \widehat{u} \dot{\xi}+4 g \bar{h}}
\end{array}\right.
$$


and so

$$
\widehat{\alpha}_{1}=\frac{\widehat{\lambda}^{2} \Delta(h \sigma)-\Delta(h \sigma(u+\dot{\xi}))}{\widehat{\lambda}^{2}-\widehat{\lambda}^{1}}, \quad \widehat{\alpha}_{2}=\frac{-\widehat{\lambda}^{1} \Delta(h \sigma)+\Delta(h \sigma(u+\dot{\xi}))}{\widehat{\lambda}^{2}-\widehat{\lambda}^{1}} .
$$

Now substitution of (4.29) and (4.31) into (4.30) gives

$$
\begin{aligned}
\widehat{\beta}_{1}= & -\frac{1}{\left(\widehat{\lambda}^{2}-\widehat{\lambda}^{1}\right)}\left(-\widehat{\lambda}^{2} \Delta(h \sigma \dot{\xi})-\left(-(\widehat{u}+\dot{\xi})^{2}+\widehat{c}^{2}\right) \Delta(h \sigma)\right. \\
& \left.-\widehat{c}^{2}(\widehat{\sigma} \Delta \beta-\Delta \sigma \bar{h})-\widehat{G}-\widehat{\lambda}^{1} \widehat{\lambda}^{2} \Delta(h \sigma)\right), \\
\widehat{\beta}_{2}= & -\frac{1}{\left(\widehat{\lambda}^{2}-\widehat{\lambda}^{1}\right)}\left(\widehat{\lambda}^{1} \Delta(h \sigma \dot{\xi})+\left(-(\widehat{u}+\dot{\xi})^{2}+\widehat{c}^{2}\right) \Delta(h \sigma)\right. \\
& \left.+\widehat{c}^{2}(\widehat{\sigma} \Delta \beta-\Delta \sigma \bar{h})+\widehat{G}+\widehat{\lambda}^{1} \widehat{\lambda}^{2} \Delta(h \sigma)\right) .
\end{aligned}
$$

The total fluctuation in the first wave family for the steady state at rest, $u=0, \Delta(\beta+h)=0$, becomes

$$
\widehat{\alpha}_{1} \widehat{\lambda}^{1}-\widehat{\beta}_{1}=-\frac{\widehat{c}^{2} \bar{\sigma}}{\widehat{\lambda}^{2}-\widehat{\lambda}^{1}} \Delta(h+\beta),
$$

and the total fluctuation in the second wave family becomes

$$
\widehat{\alpha}_{2} \widehat{\lambda}^{2}-\widehat{\beta}_{2}=\frac{\widehat{c}^{2} \bar{\sigma}}{\widehat{\lambda}^{2}-\widehat{\lambda}^{1}} \Delta(h+\beta)
$$

and so the first of (4.30), $\Delta(h u \sigma)=0$, is recovered, which insures that the steady state solutions at rest are recognized.

The shallow-water equations (4.27) can be written as

$$
\mathrm{q}_{t}+\mathbb{A}(\mathrm{q}, t) \mathrm{q}_{x}=\Psi(\mathrm{q}, x)
$$

where

$$
\mathbf{q}=\left[\begin{array}{c}
h \sigma \\
h \sigma(u+\dot{\xi})
\end{array}\right], \quad \mathbb{A}(\mathbf{q}, t)=\left[\begin{array}{cc}
0 & 1 \\
-(u+\dot{\xi})^{2}+g h & 2 u+\dot{\xi}
\end{array}\right], \quad \Psi(\mathbf{q}, x)=\left[\begin{array}{c}
\dot{\xi}(h \sigma)_{x} \\
g h^{2} \sigma_{x}-g h \sigma \beta_{x}
\end{array}\right] .
$$

Now the numerical scheme takes the general form (3.24) with right-going and left-going waves (3.25).

\section{Review of the f-wave finite volume method}

In this section we briefly review the high resolution wave propagation finite volume algorithms developed by BALE ET AL. [5, 22] and used by [5, 22, 11, 23, 12, 24, 25, 19, 18, 2, 3]. The wave propagation algorithm is Godunov type finite volume method often referred as REA algorithm, standing for reconstruction-evolve-average, making use of Riemann problems to determine the numerical update at each time step. Godunov's method uses the Riemann solutions to evaluate 
cell interface fluxes at each time step. In LeVeque's wave propagation algorithm the waves arising in Riemann solutions are re-averaged onto adjacent grid cells in order to update the numerical solution [12]. LeVeque's method is applicable to hyperbolic systems of the form (3.23). The solution to the Riemann problem consists of $m$ waves denoted by $\mathcal{W}^{p} \in \mathbb{R}^{m}$ propagating out from each grid cell interface at speeds $s^{p}$. These waves are related to the jump discontinuity at each grid cell interface via

$$
\mathbb{Q}_{i}-\mathbb{Q}_{i-1}=\sum_{p=1}^{m} \mathcal{W}_{i-1 / 2}^{p}=\sum_{p=1}^{m} \alpha_{i-1 / 2}^{p} r_{i-1 / 2}^{p},
$$

where $r_{i-1 / 2}^{p}$ are eigenvectors of the approximate flux Jacobian $\widehat{\mathbb{A}}_{i-1 / 2}$ and $\mathbb{Q}_{i}^{n}=\frac{1}{\Delta x} \int_{\mathcal{C}_{i}} \mathbf{q}\left(x, t^{n}\right) d x$ with $\mathcal{C}_{i}=\left[x_{i-1 / 2}, x_{i+1 / 2}\right], \Delta x=\left(x_{i+1 / 2}-x_{i-1 / 2}\right)$ and $\Delta t=\left(t^{n+1}-t^{n}\right)$ and the domain is partitioned into grid cells $\mathcal{C}$. This amounts to a projection of the jump in $\mathbb{Q}$ onto the eigenspace of $\widehat{\mathbb{A}}_{i-1 / 2}$. The first order upwind method then reads

$$
\mathbb{Q}_{i}^{n+1}=\mathbb{Q}_{i}^{n}-\frac{\Delta t}{\Delta x}\left(\mathbb{A}^{+} \Delta \mathbb{Q}_{i-1 / 2}+\mathbb{A}^{-} \Delta \mathbb{Q}_{i+1 / 2}\right)
$$

The fluctuations $\mathbb{A}^{ \pm} \Delta \mathbb{Q}_{i \mp 1 / 2}^{n}$ are determined by solutions to Riemann problems at the cell interfaces at $x_{i \pm 1 / 2}$. The term $\mathbb{A}^{+} \Delta \mathbb{Q}_{i-1 / 2}$ represents the net updating contribution from the rightward moving waves into grid cell $\mathcal{C}_{i}$ from the left interface, and $\mathbb{A}^{-} \Delta \mathbb{Q}_{i+1 / 2}$ represents the net updating contribution from the leftward moving waves into cell $\mathcal{C}_{i}$ from the right interface. These fluctuations can be defined in terms of waves as

$$
\mathbb{A}^{ \pm} \Delta \mathbb{Q}_{i-1 / 2}=\sum_{p=1}^{m}\left(s_{i-1 / 2}^{p}\right)^{ \pm} \mathcal{W}_{i-1 / 2}^{p},
$$

where $s_{i-1 / 2}^{+}=\max \left(s_{i-1 / 2}^{p}, 0\right)$ and $s_{i-1 / 2}^{-}=\min \left(s_{i-1 / 2}^{p}, 0\right)$. The wave propagation method (5.35) can be extended to second order accuracy using limiters applied to each wave such that

$$
\mathbb{Q}_{i}^{n+1}=\mathbb{Q}_{i}^{n}-\frac{\Delta t}{\Delta x}\left(\mathbb{A}^{+} \Delta \mathbb{Q}_{i-1 / 2}+\mathbb{A}^{-} \Delta \mathbb{Q}_{i+1 / 2}\right)-\frac{\Delta t}{\Delta x}\left(\widetilde{F}_{i+1 / 2}-\widetilde{F}_{i-1 / 2}\right),
$$

where

$$
\widetilde{F}_{i-1 / 2}=\frac{1}{2} \sum_{p=1}^{m}\left|s_{i-1 / 2}^{p}\right|\left(1-\frac{\Delta t}{\Delta x}\left|s_{i-1 / 2}^{p}\right|\right) \widetilde{\mathcal{W}}_{i-1 / 2}^{p},
$$

where $\widetilde{\mathcal{W}}_{i-1 / 2}^{p}$ are limited versions of $\mathcal{W}_{i-1 / 2}^{p}$. There are different standard limiter functions that ensure TVD stability of the solution (see $[22,12])$. A common choice for the pair $\left\{r_{i-1 / 2}^{p}, s_{i-1 / 2}^{p}\right\}$ is the $p$ th eigenpair of a local linear approximation to the flux Jacobian matrix $\widehat{\mathbb{A}}$ (q) at $x_{i-1 / 2}$ such as Roe averaging.

A consistent alternative approach to the wave propagation method (5.34) for a conservation law is to decompose the jump in fluxes into waves instead of the states q such that

$$
f\left(\mathbb{Q}_{i}\right)-f\left(\mathbb{Q}_{i-1}\right)=\sum_{p=1}^{m} \mathcal{Z}_{i-1 / 2}^{p}=\sum_{p=1}^{m} \beta_{i-1 / 2}^{p} r_{i-1 / 2}^{p},
$$


where the waves $\mathcal{Z}_{i-1 / 2}^{p}=\beta_{i-1 / 2}^{p} r_{i-1 / 2}^{p}$ are called $f$-waves and represent propagating jumps in the flux and similar to the $\mathcal{W}$ s we have $\mathcal{Z}^{p} \in \mathbb{R}^{m}$. Fluctuations are therefore defined by

$$
\mathbb{A}^{ \pm} \Delta \mathbb{Q}_{i-1 / 2}=\sum_{p=1}^{m} \operatorname{sgn}\left(s_{i-1 / 2}^{p}\right) \mathcal{Z}_{i-1 / 2}^{p} .
$$

The higher order correction terms are given by

$$
\widetilde{F}_{i-1 / 2}=\frac{1}{2} \sum_{p=1}^{m} \operatorname{sgn}\left(s_{i-1 / 2}^{p}\right)\left(1-\frac{\Delta t}{\Delta x}\left|s_{i-1 / 2}^{p}\right|\right) \widetilde{\mathcal{Z}}_{i-1 / 2}^{p},
$$

where $\widetilde{\mathcal{Z}}_{i-1 / 2}^{p}$ is a limited version of the f-wave $\mathcal{Z}_{i-1 / 2}^{p}$ using a TVD limiter [22].

The advantage of using the f-wave method over the wave-propagation method is that it is conservative regardless of the linearization used for the flux Jacobian to calculate the eigenspace. It also extends to spatially varying flux terms $\Psi(\mathrm{q}, x)$. The advantage that we will make the most use of in solving the shallow-water equations over variable topography and cross-section is the ability to use source terms to modify the flux difference before doing wave decomposition such that

$$
\delta=f\left(\mathbb{Q}_{i}\right)-f\left(\mathbb{Q}_{i-1}\right)-\Delta x \Psi_{i-1 / 2}=\sum_{p=1}^{m} \mathcal{Z}_{i-1 / 2}^{p},
$$

for some representation of the source term $\Psi$ at $x_{i-1 / 2}$ such that

$$
\Delta x \Psi_{i-1 / 2} \approx \int \Psi(\mathrm{q}, x) d x
$$

The basic steps in implementing the f-wave-propagation method are the evaluation of the relevant states to the Riemann problem using the vectors $\mathbb{Q}_{i}$ and $\mathbb{Q}_{i-1}$, computation of the eigenvalues and eigenvectors $\left(s^{p}, r^{p}\right)$, computation of the jump in the fluxes and source terms $\delta$, projection of $\delta$ onto the eigenspace to determine the f-waves $\mathcal{Z}^{p}$, and finally calculation of the fluctuations $\mathbb{A}^{ \pm} \Delta \mathbb{Q}$.

\section{New augmented f-wave Riemann solvers}

In this section the augmented Riemann finite volume solvers first developed in $[12,11]$ are adapted for the shallow-water equations over variable topography and variable cross-section (1.1). The motivation for developing the augmented Riemann solvers as discussed in [12] is that it unifies desirable features of different finite volume solvers such as the Roe solver [26, 27], HLLE-type solvers [8, 9], and the f-wave-propagation approach [5]. It is known that Roe-type solvers require entropy fixes to prevent non-physical solutions, and fail to preserve depth nonnegativity for shallow-water equations with a very thin layer of fluid. An inherent weakness of the f-wave-propagation method for the shallow-water equations is that it is not clear how to preserve positivity in the Riemann solution [12] which is an important property to simulate sloshing with wetting and drying. And this is because the depth or wetted cross-section of the vessel is not included in the jump decomposition. Also wave-propagation type solvers may 
inaccurately resolve a true Riemann solution if the solution contains a transonic rarefaction that spreads in both directions. The HLLE solver $[8,9]$ preserves depth positivity. However, HLLtype solvers are not well-balanced, and require modifications in order to preserve steady states for the shallow-water equations [12, 23].

The advantages of the augmented Riemann solvers are: preserving depth positivity, providing an exact solution for a single shock Riemann problem, having a natural entropy fix, and being well-balanced in the sense that it preserves a large class of steady states even non-stationary steady states with non-zero fluid velocity [12].

\subsection{An augmented solver}

The hyperbolic shallow water system (1.1) can be augmented by considering a governing equation for the flux of the momentum equation which is

$$
\left(h u^{2} \sigma+\frac{1}{2} g h^{2} \sigma\right)_{t}+\left(-u^{2}+g h\right)(h u \sigma)_{x}+2 u\left(h u^{2} \sigma+\frac{1}{2} g h^{2} \sigma\right)_{x}=2 u\left(\frac{1}{2} g h^{2} \sigma_{x}-g h \sigma \beta_{x}\right) .
$$

As is known the standard fractional step approach to include source terms into numerical solutions fails at preserving the steady states (see [5, 21, 11]). Instead the source terms can be treated by defining additional waves into the jump decomposition at grid cell interface. This can be done by considering additional state variables, bottom topography $\beta(x)$ and cross-section function $\sigma(x)$, in the state vector. So by including equation (6.41) and augmented variables $\beta$ and $\sigma$, the inhomogeneous hyperbolic system (1.1) can be written as an overdetermined augmented homogeneous system

$$
\widetilde{\mathrm{q}}_{t}+\widetilde{\mathbb{A}}(\widetilde{\mathrm{q}}) \widetilde{\mathrm{q}}_{x}=0
$$

where

$$
\widetilde{\mathbf{q}}=\left[\begin{array}{c}
h \sigma \\
h u \sigma \\
h u^{2} \sigma+\frac{1}{2} g h^{2} \sigma \\
\beta \\
\sigma
\end{array}\right], \quad \widetilde{\mathbb{A}}(\widetilde{\mathbf{q}})=\left[\begin{array}{ccccc}
0 & 1 & 0 & 0 & 0 \\
-u^{2}+g h & 2 u & 0 & g h \sigma & -g h^{2} \\
0 & -u^{2}+g h & 2 u & 2 u g h \sigma & -g h^{2} u \\
0 & 0 & 0 & 0 & 0 \\
0 & 0 & 0 & 0 & 0
\end{array}\right] .
$$

And hence instead of the decompositions (5.34) or (5.37), decompositions of the following form can be considered

$$
\left[\begin{array}{c}
(h \sigma)_{i}-(h \sigma)_{i-1} \\
(h u \sigma)_{i}-(h u \sigma)_{i-1} \\
\left(h u^{2} \sigma+\frac{1}{2} g h^{2} \sigma\right)_{i}-\left(h u^{2} \sigma+\frac{1}{2} g h^{2} \sigma\right)_{i-1} \\
\beta_{i}-\beta_{i-1} \\
\sigma_{i}-\sigma_{i-1}
\end{array}\right]=\sum_{p=1}^{5} \alpha_{i-1 / 2}^{p} w_{i-1 / 2}^{p}
$$

where $w_{i-1 / 2}^{p} \in \mathbb{R}^{5}$, and $\beta_{i}$ and $\beta_{i-1}$ are cell-averaged approximations to the bottom topography, and $\sigma_{i}$ and $\sigma_{i-1}$ are cell-averaged approximations to the cross-section function in $\mathcal{C}_{i}$ and $\mathcal{C}_{i-1}$. 
The matrix $\widetilde{\mathbb{A}}(\widetilde{\mathrm{q}})$ in $(6.43)$ has the eigenvectors

$$
\begin{aligned}
& r^{1}(\widetilde{\mathbf{q}})=\left[\begin{array}{c}
1 \\
\lambda^{1}(\widetilde{\mathbf{q}}) \\
\lambda^{1}(\widetilde{\mathbf{q}})^{2} \\
0 \\
0
\end{array}\right], \quad r^{2}(\widetilde{\mathbf{q}})=\left[\begin{array}{l}
0 \\
0 \\
1 \\
0 \\
0
\end{array}\right], \quad r^{3}(\widetilde{\mathbf{q}})=\left[\begin{array}{c}
1 \\
\lambda^{3}(\widetilde{\mathbf{q}}) \\
\lambda^{3}(\widetilde{\mathbf{q}})^{2} \\
0 \\
0
\end{array}\right], \quad r^{4}(\widetilde{\mathbf{q}})=\left[\begin{array}{c}
g h \sigma-g h^{2} \\
\lambda^{1}(\widetilde{\mathbf{q}}) \lambda^{3}(\widetilde{\mathbf{q}}) \\
0 \\
\frac{1}{2} g h^{2}-g h \sigma \\
1 \\
1
\end{array}\right], \\
& r^{5}(\widetilde{\mathbf{q}})=r^{4}(\widetilde{\mathbf{q}}),
\end{aligned}
$$

with associated eigenvalues

$$
\lambda^{1}(\widetilde{\mathbf{q}})=u-\sqrt{g h}, \quad \lambda^{2}(\widetilde{\mathbf{q}})=2 u, \quad \lambda^{3}(\widetilde{\mathbf{q}})=u+\sqrt{g h}, \quad \lambda^{4}(\widetilde{\mathbf{q}})=\lambda^{5}(\widetilde{\mathbf{q}})=0 .
$$

In order to ensure conservation, regardless of the form of $w_{i-1 / 2}^{p}$, the second and third components of the decomposition (6.44) are used to define the f-waves $\mathcal{Z}_{i-1 / 2}^{p} \in \mathbb{R}^{2}$, for $p=1, \ldots, 5$. The fluctuations are defined as

$$
\mathbb{A}^{ \pm} \Delta \mathbb{Q}_{i-1 / 2}=\sum_{p=1}^{5} \operatorname{sgn}\left(s_{i-1 / 2}^{p}\right) \mathcal{Z}_{i-1 / 2}^{p},
$$

noting that the waves corresponding to $p=4,5$ are not included in the fluctuations since eigenspeeds are zero as given in (6.46). The effect of the geometric source terms are included in this construction of the fluctuations by a proper choice of $w_{i-1 / 2}^{4}$ and $w_{i-1 / 2}^{5}$ as will be described shortly.

We choose the vectors $w_{i-1 / 2}^{p} \in \mathbb{R}^{5}$ and associated wave speeds $s_{i-1 / 2}^{p}$, for $p=1, \ldots, 3$ as follows

$$
\begin{aligned}
& \left\{w_{i-1 / 2}^{1}, s_{i-1 / 2}^{1}\right\}=\left\{\left(1, s_{\mathcal{E}_{i-1 / 2}}^{1},\left(s_{\mathcal{E}_{i-1 / 2}}^{1}\right)^{2}, 0,0\right)^{T}, s_{\left.\mathcal{E}_{i-1 / 2}\right\}}^{1},\right. \\
& \left\{w_{i-1 / 2}^{2}, s_{i-1 / 2}^{2}\right\}=\left\{(0,0,1,0,0)^{T}, \frac{1}{2}\left(s_{\mathcal{E}_{i-1 / 2}}^{1}+s_{\mathcal{E}_{i-1 / 2}}^{3}\right)\right\}, \\
& \left\{w_{i-1 / 2}^{3}, s_{i-1 / 2}^{3}\right\}=\left\{\left(1, s_{\mathcal{E}_{i-1 / 2}}^{3},\left(s_{\mathcal{E}_{i-1 / 2}}^{3}\right)^{2}, 0,0\right)^{T}, s_{\mathcal{E}_{i-1 / 2}}^{3}\right\},
\end{aligned}
$$

where the eigenspeeds are defined by

$$
\begin{aligned}
& s_{\mathcal{E}_{i-1 / 2}}^{1}=\min \left(\lambda^{1}\left(\mathbb{Q}_{i-1}^{n}\right), \widehat{\lambda}_{i-1 / 2}^{1}\right), \\
& s_{\mathcal{E}_{i-1 / 2}}^{3}=\max \left(\lambda^{3}\left(\mathbb{Q}_{i}^{n}\right), \widehat{\lambda}_{i-1 / 2}^{3}\right),
\end{aligned}
$$

where $\widehat{\lambda}_{i-1 / 2}^{1}=\widehat{u}-\widehat{c}$ and $\widehat{\lambda}_{i-1 / 2}^{3}=\widehat{u}+\widehat{c}$ are the Roe eigenvalues with $\widehat{u}$ and $\widehat{C}$ as defined in $\S 3$. The eigenspeeds (6.49) are called the Einfeldt speeds as first suggested by Einfeldt in the HLLE solver $[8,9,16]$ in order to preserve depth positivity [12]. As is discussed in $[11,12]$ the pair $\left\{w_{i-1 / 2}^{2}, s_{i-1 / 2}^{2}\right\}$, the corrector wave, can be chosen in various ways in order to have the correct eigenspeeds for the Riemann problems with a strong rarefaction or with a wet/dry front. See [12] for a detailed discussion.

The pairs $\left\{w_{i-1 / 2}^{4}, s_{i-1 / 2}^{4}\right\}$ and $\left\{w_{i-1 / 2}^{5}, s_{i-1 / 2}^{5}\right\}$ which are related to the steady state waves are given below. 


\subsection{The steady state waves}

In this section we present an adapted version of the steady state wave due to the bathymetry function given in $[11,12]$ to incorporate both the effects of the bottom topography and wetted cross-section, in the jump decompositions.

Incorporation of the bottom surface $\beta(x)$ and the cross-section function $\sigma(x)$ in the state vector $\widetilde{q}$ introduces two new linearly degenerate fields with identically zero eigenvalues. As is discussed in [11] these fields carry the variation of $\widetilde{q}$ for smooth steady states, since the nontrivial steady state solutions to (6.42) satisfy

$$
\widetilde{\mathbb{A}}(\widetilde{\mathrm{q}}) \widetilde{\mathrm{q}}_{x}=0,
$$

implying that $\widetilde{\mathrm{q}}_{x}$ is proportional to $r^{4}(\widetilde{\mathrm{q}})$ and $r^{5}(\widetilde{\mathrm{q}})$. Meaning that the smooth steady state solutions $\widetilde{\mathrm{q}}$ are integral curves of $r^{4}(\widetilde{\mathrm{q}})$ and $r^{5}(\widetilde{\mathrm{q}})$ in $\mathbb{R}^{5}$, parameterized by $x$. For the augmented solver (6.44) the pairs $\left\{w_{i-1 / 2}^{4}, s_{i-1 / 2}^{4}\right\}$ and $\left\{w_{i-1 / 2}^{5}, s_{i-1 / 2}^{5}\right\}$ are chosen to be approximations to $r^{4}(\widetilde{\mathrm{q}}), \lambda^{4}(\widetilde{\mathrm{q}}), r^{5}(\widetilde{\mathrm{q}})$, and $\lambda^{5}(\widetilde{\mathrm{q}})$ based on the local solutions. Since the eigenspeeds are zero, the resulting stationary waves in the approximate Riemann solution are jump discontinuities remaining at the cell interface which are contributions by the source terms. So the strategy is to define the vectors $w_{i-1 / 2}^{4}$ and $w_{i-1 / 2}^{5}$ appropriately using special averages of the states $\mathbb{Q}_{i}$ and $\mathbb{Q}_{i-1}$ which is believed then the solver preserves a larger class of steady states [12].

The required forms of the eigenvectors $w_{i-1 / 2}^{4}$ and $w_{i-1 / 2}^{5}$ evaluated with special averages of the right and left states are given in the following theorem:

Theorem 1. Suppose that a smooth steady state solution to the shallow-water equations exists between two points, $x_{l}$ and $x_{r}$, with $\beta\left(x_{l}\right) \neq \beta\left(x_{r}\right)$ and $\sigma\left(x_{l}\right) \neq \sigma\left(x_{r}\right)$. If the vector $\widetilde{\mathrm{q}}(x, t) \in$ $\mathbb{R}^{5}$ is differenced between $x_{l}$ and $x_{r}$, then the difference satisfies

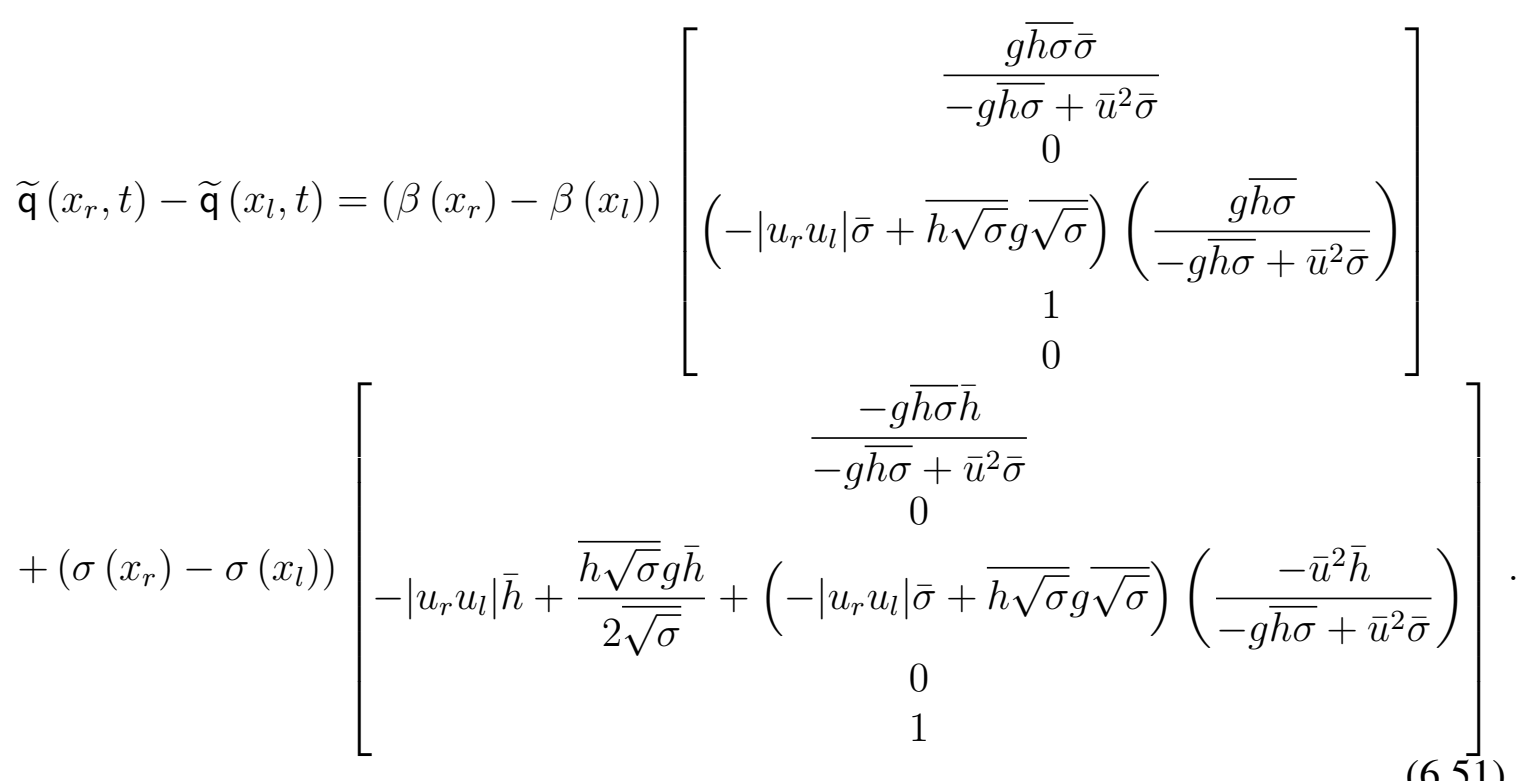

Proof of Theorem 1. The subscripts ()$_{r}$ and $(\quad)_{l}$ are used to denote the evaluation of variables at $x_{r}$ and $x_{l}$, respectively. And $\Delta$ is used to denote differencing such quantities between $x_{r}$ and $x_{l}$. Consider a smooth steady state between two points, $x_{l}$ and $x_{r}$, surrounding varying topography and varying cross-section. For all smooth steady states $(h u \sigma)_{x} \equiv 0$, and hence

$$
\Delta(h u \sigma)=0 .
$$


Using this fact the difference in the momentum flux can be related as

$$
\begin{aligned}
\Delta\left(h u^{2} \sigma+\frac{1}{2} g h^{2} \sigma\right) & =\left(\frac{(h u \sigma)_{r}^{2}}{(h \sigma)_{r}}-\frac{(h u \sigma)_{l}^{2}}{(h \sigma)_{l}}\right)+\frac{g}{2}\left(h_{r} \sqrt{\sigma_{r}}+h_{l} \sqrt{\sigma_{l}}\right)\left(h_{r} \sqrt{\sigma_{r}}-h_{l} \sqrt{\sigma_{l}}\right), \\
& =\left(\frac{\left|h_{r} u_{r} \sigma_{r} h_{l} u_{l} \sigma_{l}\right|}{h_{r} \sigma_{r}}-\frac{h_{r} u_{r} \sigma_{r} h_{l} u_{l} \sigma_{l}}{h_{l} \sigma_{l}}\right)+\frac{g}{2}\left(h_{r} \sqrt{\sigma_{r}}+h_{l} \sqrt{\sigma_{l}}\right)\left(h_{r} \sqrt{\sigma_{r}}-h_{l} \sqrt{\sigma_{l}}\right), \\
& =\left(h_{l} \sigma_{l}\left|u_{r} u_{l}\right|-h_{r} \sigma_{r}\left|u_{r} u_{l}\right|\right)+\frac{g}{2}\left(h_{r} \sqrt{\sigma_{r}}+h_{l} \sqrt{\sigma_{l}}\right)\left(h_{r} \sqrt{\sigma_{r}}-h_{l} \sqrt{\sigma_{l}}\right), \\
& =-\left|u_{r} u_{l}\right| \Delta(h \sigma)+g \overline{h \sqrt{\sigma}} \Delta(h \sqrt{\sigma}), \\
& =-\left|u_{r} u_{l}\right|(\bar{h} \Delta \sigma+\bar{\sigma} \Delta h)+g \overline{h \sqrt{\sigma}}(\bar{h} \Delta \sqrt{\sigma}+\overline{\sqrt{\sigma}} \Delta h), \\
& =-\left|u_{r} u_{l}\right|(\bar{h} \Delta \sigma+\bar{\sigma} \Delta h)+g \overline{h \sqrt{\sigma}}\left(\frac{\bar{h}}{2 \overline{\sqrt{\sigma}}} \Delta \sigma+\overline{\sqrt{\sigma}} \Delta h\right) .
\end{aligned}
$$

We can relate $\Delta h$ to $\Delta \sigma$ and $\Delta \beta$. Note that for a steady state

$$
\left(h u^{2} \sigma+\frac{1}{2} g h^{2} \sigma\right)_{x}+g h \sigma \beta_{x}-\frac{1}{2} g h^{2} \sigma_{x}=0,
$$

which can be transformed into

$$
\left(\frac{1}{2} u^{2}+g h+g \beta\right)_{x}=0 .
$$

The discrete form of (6.53) becomes

$$
-g \Delta h-g \Delta \beta=\Delta\left(\frac{1}{2} u^{2}\right)
$$

or

$$
\begin{aligned}
-g \overline{h \sigma} \Delta h-g \overline{h \sigma} \Delta \beta & =\left(\frac{h_{r} \sigma_{r}+h_{l} \sigma_{l}}{2}\right)\left(\frac{u_{r}^{2}-u_{l}^{2}}{2}\right) \\
& =\frac{1}{4}\left[h_{r} u_{r}^{2} \sigma_{r}-h_{r} u_{l}^{2} \sigma_{r}+h_{l} u_{r}^{2} \sigma_{l}-h_{l} u_{l}^{2} \sigma_{l}\right] \\
& =\frac{1}{4}\left[\left(2 h_{r} u_{r}^{2} \sigma_{r}-h_{r} u_{r}^{2} \sigma_{r}\right)+h_{l} u_{r}^{2} \sigma_{l}-h_{r} u_{l}^{2} \sigma_{r}-\left(2 h_{l} u_{l}^{2} \sigma_{l}-h_{l} u_{l}^{2} \sigma_{l}\right)\right] \\
& =\frac{1}{4}\left[\left(2 h_{l} u_{l} \sigma_{l} u_{r}-h_{r} u_{r}^{2} \sigma_{r}\right)+h_{l} u_{r}^{2} \sigma_{l}-h_{r} u_{l}^{2} \sigma_{r}-\left(2 h_{r} u_{r} \sigma_{r} u_{l}-h_{l} u_{l}^{2} \sigma_{l}\right)\right] \\
& =\frac{1}{4}\left[\left(h_{l} u_{l}^{2} \sigma_{l}+2 h_{l} u_{l} \sigma_{l} u_{r}+h_{l} u_{r}^{2} \sigma_{l}\right)-\left(h_{r} u_{l}^{2} \sigma_{r}+2 h_{r} u_{r} \sigma_{r} u_{l}+h_{r} u_{r}^{2} \sigma_{r}\right)\right] \\
& =\frac{1}{4}\left[h_{l} \sigma_{l}\left(u_{r}+u_{l}\right)^{2}-h_{r} \sigma_{r}\left(u_{r}+u_{l}\right)^{2}\right] \\
& =-\bar{u}^{2} \Delta(h \sigma) \\
& =-\bar{u}^{2}(\bar{h} \Delta \sigma+\bar{\sigma} \Delta h)
\end{aligned}
$$

and so

$$
\Delta h=\left(\frac{-\bar{u}^{2} \bar{h}}{-g \overline{h \sigma}+\bar{u}^{2} \bar{\sigma}}\right) \Delta \sigma+\left(\frac{g \overline{h \sigma}}{-g \overline{h \sigma}+\bar{u}^{2} \bar{\sigma}}\right) \Delta \beta .
$$


Note that equation (6.54) recovers the motionless steady state $(h+\beta)_{x} \equiv 0$. Now substituting (6.54) into (6.52) gives

$$
\begin{aligned}
\Delta\left(h u^{2} \sigma+\frac{1}{2} g h^{2} \sigma\right)= & \left(-\left|u_{r} u_{l}\right| \bar{\sigma}+\overline{h \sqrt{\sigma} g \overline{\sqrt{\sigma}}}\right)\left(\frac{g \overline{h \sigma}}{-g \overline{h \sigma}+\bar{u}^{2} \bar{\sigma}}\right) \Delta \beta \\
& +\left(-\left|u_{r} u_{l}\right| \bar{h}+\frac{\overline{h \sqrt{\sigma} g \bar{h}}}{2 \overline{\sqrt{\sigma}}}+\left(-\left|u_{r} u_{l}\right| \bar{\sigma}+\overline{h \sqrt{\sigma}} g \overline{\sqrt{\sigma}}\right)\left(\frac{-\bar{u}^{2} \bar{h}}{-g \overline{h \sigma}+\bar{u}^{2} \bar{\sigma}}\right)\right) \Delta \sigma,
\end{aligned}
$$

which recovers the second components of the steady state vecotrs in (6.51). Also note that

$$
\begin{aligned}
\Delta(h \sigma) & =\bar{h} \Delta \sigma+\bar{\sigma} \Delta h \\
& =\left(\frac{g \overline{h \sigma} \bar{\sigma}}{-g \overline{h \sigma}+\bar{u}^{2} \bar{\sigma}}\right) \Delta \beta+\left(\frac{-g \overline{h \sigma} \bar{h}}{-g \overline{h \sigma}+\bar{u}^{2} \bar{\sigma}}\right) \Delta \sigma,
\end{aligned}
$$

which recovers the first components of the steady state vectors in (6.51).

Note that (6.51) recovers Theorem 1 in [12] for a vessel with $\sigma(x)=1$.

Theorem 1 implies that if the numerical data, $\widetilde{\mathbb{Q}}_{i, i-1} \in \mathbb{R}^{5}$ with

$$
\widetilde{\mathbb{Q}}_{i}=\left(h \sigma, h u \sigma, h u^{2} \sigma+\frac{1}{2} g h^{2} \sigma, \beta, \sigma\right)_{i}^{T},
$$

correspond to evaluating a smooth steady state at two points surrounding variable topography and cross-section, then the decomposition (6.44) will have only two nonzero waves, steady state waves, since

$$
\widetilde{\mathbb{Q}}_{i}-\widetilde{\mathbb{Q}}_{i-1}=\alpha_{i-1 / 2}^{4} w_{i-1 / 2}^{4}+\alpha_{i-1 / 2}^{5} w_{i-1 / 2}^{5}=\left(\beta_{i}-\beta_{i-1}\right) w_{i-1 / 2}^{4}+\left(\sigma_{i}-\sigma_{i-1}\right) w_{i-1 / 2}^{5} .
$$

Since the steady state wave is stationary the discrete steady state will be exactly maintained as a jump discontinuity at the cell interface $x_{i-1 / 2}$ [12]. Theorem 1 or equation (6.57) imply that for any two points $\widetilde{\mathbb{Q}}_{i} \in \mathbb{R}^{5}$ and $\widetilde{\mathbb{Q}}_{i-1} \in \mathbb{R}^{5}$ lying on integral curves of $r^{4}(\widetilde{\mathrm{q}}) \in \mathbb{R}^{5}$ and $r^{5}(\widetilde{\mathrm{q}}) \in$ $\mathbb{R}^{5}$, then those points are connected by the vectors $\left(\beta_{i}-\beta_{i-1}\right) w_{i-1 / 2}^{4}$ and $\left(\sigma_{i}-\sigma_{i-1}\right) w_{i-1 / 2}^{5}$, respectively. And the steady state vectors are

$$
w_{i-1 / 2}^{4}=\left(\frac{g \overline{h \sigma} \bar{\sigma}}{-g \overline{h \sigma}+\bar{u}^{2} \bar{\sigma}}, 0,\left(-\left|u_{r} u_{l}\right| \bar{\sigma}+\overline{h \sqrt{\sigma}} g \overline{\sqrt{\sigma}}\right)\left(\frac{g \overline{h \sigma}}{-g \overline{h \sigma}+\bar{u}^{2} \bar{\sigma}}\right), 1,0\right)^{T}
$$

and

$$
w_{i-1 / 2}^{5}=\left(\frac{-g \bar{h} \bar{h}}{-g \overline{h \sigma}+\bar{u}^{2} \bar{\sigma}}, 0,-\left|u_{r} u_{l}\right| \bar{h}+\frac{\overline{h \sqrt{\sigma} g \bar{h}}}{2 \overline{\sqrt{\sigma}}}+\left(-\left|u_{r} u_{l}\right| \bar{\sigma}+\overline{h \sqrt{\sigma} g \overline{\sqrt{\sigma}}}\right)\left(\frac{-\bar{u}^{2} \bar{h}}{-g \overline{h \sigma}+\bar{u}^{2} \bar{\sigma}}\right), 0,1\right)^{T} .
$$

The discontinuities proportional to (6.58) and (6.59) at the cell interface act as a source of momentum. The stationary waves $\mathcal{Z}_{i-1 / 2}^{4}$ and $\mathcal{Z}_{i-1 / 2}^{5}$ are not included in the fluctuations (6.47) since these waves do not move into either adjacent grid cell. Therefore, the decomposition 
(6.44) defines exactly the same fluctuations as would be obtained by first subtracting the stationary waves from the Riemann data

$$
\left[\begin{array}{c}
(h \sigma)_{i}-(h \sigma)_{i-1} \\
(h u \sigma)_{i}-(h u \sigma)_{i-1} \\
\left(h u^{2} \sigma+\frac{1}{2} g h^{2} \sigma\right)_{i}-\left(h u^{2} \sigma+\frac{1}{2} g h^{2} \sigma\right)_{i-1} \\
\beta_{i}-\beta_{i-1} \\
\sigma_{i}-\sigma_{i-1}
\end{array}\right]-\alpha_{i-1 / 2}^{4} w_{i-1 / 2}^{4}-\alpha_{i-1 / 2}^{5} w_{i-1 / 2}^{5}=\sum_{p=1}^{3} \alpha_{i-1 / 2}^{p} w_{i-1 / 2}^{p}
$$

where $\alpha_{i-1 / 2}^{4}=\left(\beta_{i}-\beta_{i-1}\right)$ and $\alpha_{i-1 / 2}^{5}=\left(\sigma_{i}-\sigma_{i-1}\right)$. Equation (6.60) represents subtracting an approximation to the geometric source terms from the momentum flux. By using $\left(w_{i-1 / 2}^{4}\right)^{3}$ and $\left(w_{i-1 / 2}^{5}\right)^{3}$ to approximate $-g h \sigma$ and $\frac{1}{2} g h^{2}$, the smooth steady states over variable bottom topography and variable cross-section are preserved. That is,

$$
\begin{aligned}
& \left(\beta\left(x_{r}\right)-\beta\left(x_{l}\right)\right)\left(w_{i-1 / 2}^{4}\left(\mathbf{q}\left(x_{l}, t\right), \mathbf{q}\left(x_{r}, t\right)\right)\right)^{3}+\left(\sigma\left(x_{r}\right)-\sigma\left(x_{l}\right)\right)\left(w_{i-1 / 2}^{5}\left(\mathbf{q}\left(x_{l}, t\right), \mathbf{q}\left(x_{r}, t\right)\right)\right)^{3} \\
& =-\int_{x_{l}}^{x_{r}} g h \sigma \beta_{x} d x+\int_{x_{l}}^{x_{r}} \frac{1}{2} g h^{2} \sigma_{x} d x
\end{aligned}
$$

is exactly satisfied for such solutions, as long as $\beta\left(x_{r}\right)-\beta\left(x_{l}\right) \neq 0$ and $\sigma\left(x_{r}\right)-\sigma\left(x_{l}\right) \neq 0$.

\subsection{Preserving depth positivity with the geometric source terms}

It is known that for the pure shallow-water equations, the HLLE-type solvers preserve middle state depth, the Einfeldt depth $h_{\mathcal{E}}^{\star}$, by approximating the Riemann solution with two simple waves propagating with predetermined Einfeldt speeds $s_{\mathcal{E}}^{1}$ and $s_{\mathcal{E}}^{3}$. For the shallow-water equations (1.1) the middle state wetted cross-section, $(h \sigma)_{\mathcal{E}}^{\star}$, in the absence of the source terms, can be obtained by solving the splitting (6.44) with $p=1, \ldots, 3$, resulting in

$$
(h \sigma)_{\mathcal{E}}^{\star}=\frac{(h u \sigma)_{l}-(h u \sigma)_{r}+s_{\mathcal{E}}^{3}(h \sigma)_{r}-s_{\mathcal{E}}^{1}(h \sigma)_{l}}{s_{\mathcal{E}}^{3}-s_{\mathcal{E}}^{1}} .
$$

It can be proved that $(h \sigma)_{\mathcal{E}}^{\star}$ is always non-negative. The denominator in (6.62) is always positive, since the Roe speeds are such that $\widehat{\lambda}^{3}\left(q_{l}, q_{r}\right)>\widehat{\lambda}^{1}\left(q_{l}, q_{r}\right)$ for any states $q_{l}$ and $q_{r}$. For the numerator,

$$
\begin{aligned}
\left(s_{\mathcal{E}}^{3}-s_{\mathcal{E}}^{1}\right)(h \sigma)_{\mathcal{E}}^{\star} & =(h u \sigma)_{l}-(h u \sigma)_{r}+s_{\mathcal{E}}^{3}(h \sigma)_{r}-s_{\mathcal{E}}^{1}(h \sigma)_{l}, \\
& \geq(h u \sigma)_{l}-(h u \sigma)_{r}+\left(u_{r}+\sqrt{g h_{r}}\right)(h \sigma)_{r}-\left(u_{l}-\sqrt{g h_{l}}\right)(h \sigma)_{l}, \\
& =(h \sigma)_{r} \sqrt{g h_{r}}+(h \sigma)_{l} \sqrt{g h_{l}} \geq 0 .
\end{aligned}
$$

When variable bottom topography and variable cross-section are introduced into the Riemann problem, a stationary discontinuity in wetted cross-section is present in the Riemann solution at the cell interface, due to the first components of $w_{i-1 / 2}^{4}$ and $w_{i-1 / 2}^{5}$. Subtracting the source terms may result in two different middle wetted cross-section states, $(h \sigma)_{l}^{\star}$ and $(h \sigma)_{r}^{\star}$. And so 
the jump discontinuity may produce a positive cross-section to one side of the interface and a negative cross-section to the other. So it is necessary to bound the sum of the first components of $w_{i-1 / 2}^{4}$ and $w_{i-1 / 2}^{5}$. Consider the decomposition (6.44) with the associated waves (6.48), (6.58) and (6.59). Since the second components of $w_{i-1 / 2}^{2}, w_{i-1 / 2}^{4}$ and $w_{i-1 / 2}^{5}$ are zero, conservation of mass is ensured and so the middle cross-section states, $(h \sigma)_{l}^{\star}$ and $(h \sigma)_{r}^{\star}$, can be related to the single conservative middle cross-section state, $(h \sigma)_{\mathcal{E}}^{\star}$, that would arise if the source terms were absent. For a subsonic problem, where $s_{\mathcal{E}}^{1}<0<s_{\mathcal{E}}^{3}$, conservation of mass requires

$$
s_{\mathcal{E}}^{3}\left((h \sigma)_{r}-(h \sigma)_{\mathcal{E}}^{\star}\right)+s_{\mathcal{E}}^{1}\left((h \sigma)_{\mathcal{E}}^{\star}-(h \sigma)_{l}\right)=s_{\mathcal{E}}^{3}\left((h \sigma)_{r}-(h \sigma)_{r}^{\star}\right)+s_{\mathcal{E}}^{1}\left((h \sigma)_{l}^{\star}-(h \sigma)_{l}\right) .
$$

By either increasing $(h \sigma)_{l}^{\star}$ or $(h \sigma)_{r}^{\star}$, and decreasing the other, the following bounds for the maximum of the left and right wetted cross-section states can be found

$$
(h \sigma)_{r}^{\star}=\frac{\left(s_{\mathcal{E}}^{3}-s_{\mathcal{E}}^{1}\right)}{s_{\mathcal{E}}^{3}}(h \sigma)_{\mathcal{E}}^{\star},
$$

when $(h \sigma)_{l}^{\star}=0$, and

$$
(h \sigma)_{l}^{\star}=\frac{\left(s_{\mathcal{E}}^{3}-s_{\mathcal{E}}^{1}\right)}{-s_{\mathcal{E}}^{1}}(h \sigma)_{\mathcal{E}}^{\star},
$$

when $(h \sigma)_{r}^{\star}=0$. And so by preventing $(h \sigma)_{l}^{\star}$ and $(h \sigma)_{r}^{\star}$ from becoming negative then (6.63) and (6.64) imply that

$$
\frac{\left(s_{\mathcal{E}}^{3}-s_{\mathcal{E}}^{1}\right)}{s_{\mathcal{E}}^{1}}(h \sigma)_{\mathcal{E}}^{\star} \leq(h \sigma)_{r}^{\star}-(h \sigma)_{l}^{\star} \leq \frac{\left(s_{\mathcal{E}}^{3}-s_{\mathcal{E}}^{1}\right)}{s_{\mathcal{E}}^{3}}(h \sigma)_{\mathcal{E}}^{\star} .
$$

But for the splitting (6.44)

$$
(h \sigma)_{r}^{\star}-(h \sigma)_{l}^{\star}=\Delta \beta\left(w_{i-1 / 2}^{4}\right)^{1}+\Delta \sigma\left(w_{i-1 / 2}^{5}\right)^{1}
$$

and hence (6.65) would be satisfied if

$$
\frac{\left(s_{\mathcal{E}}^{3}-s_{\mathcal{E}}^{1}\right)}{s_{\mathcal{E}}^{1}}(h \sigma)_{\mathcal{E}}^{\star} \leq\left(\frac{g \overline{h \sigma}}{-g \overline{h \sigma}+\bar{u}^{2} \bar{\sigma}}\right)(\Delta \beta \bar{\sigma}-\Delta \sigma \bar{h}) \leq \frac{\left(s_{\mathcal{E}}^{3}-s_{\mathcal{E}}^{1}\right)}{s_{\mathcal{E}}^{3}}(h \sigma)_{\mathcal{E}}^{\star} .
$$

For a supersonic problem, where $0<s_{\mathcal{E}}^{1}<s_{\mathcal{E}}^{3}$ or $s_{\mathcal{E}}^{1}<s_{\mathcal{E}}^{3}<0$, bounds on $\Delta \beta\left(w_{i-1 / 2}^{4}\right)^{1}+$ $\Delta \sigma\left(w_{i-1 / 2}^{5}\right)^{1}$ are not necessary at the first order updates. Since all of the waves move in the same direction, the numerical effect of a negative middle state cross-section would be balanced by a positive one.

The augmented solver (6.44) works well for the first-order updates with variable topography, variable cross-section and dry states. However addition of second-order correction terms (5.39) may cause non-physical negative depths when dry states are present. Since interface fluxes affect more than one cell, it is necessary to limit the gross outward mass fluxes even when the net flux does not generate a negative depth [11]. An additional limiting procedure can be applied to $\widetilde{F}_{i-1 / 2}$ to maintain positivity [23]. The new limiting procedure is best illustrated by writing the second-order method (5.36) as the sum of the first-order Godunov update [23]

$$
\mathbb{Q}_{i}^{*}=\mathbb{Q}_{i}^{n}-\frac{\Delta t}{\Delta x}\left(\mathbb{A}^{+} \Delta \mathbb{Q}_{i-1 / 2}+\mathbb{A}^{-} \Delta \mathbb{Q}_{i+1 / 2}\right)
$$


and the second order correction fluxes

$$
\mathbb{Q}_{i}^{n+1}=\mathbb{Q}_{i}^{\star}-\frac{\Delta t}{\Delta x}\left(\widetilde{F}_{i+1 / 2}-\widetilde{F}_{i-1 / 2}\right)
$$

The correction flux $\widetilde{F}_{i-1 / 2}$ at a cell interface takes mass away from one cell and adds mass to the adjacent cell, with direction depending on the sign of its first component. The total gross mass flux out of the cell $\mathcal{C}_{i}$ due to the correction fluxes is then [23]

$$
\mathcal{M}_{i}=\left(\max \left(0, \widetilde{F}_{i+1 / 2}^{1}\right)-\min \left(0, \widetilde{F}_{i-1 / 2}^{1}\right)\right) \text {. }
$$

If $\Delta t \mathcal{M}_{i}$ is larger than the amount of mass remaining in the cell after first-order Godunov update, $\Delta x\left(\mathbb{Q}_{i}^{*}\right)^{1}$, then the correction fluxes could create negative depth in this cell [23]. And this can be prevented by re-limiting $\widetilde{F}_{i-1 / 2}$ based on which cell they take mass away from

$$
\widetilde{F}_{i-1 / 2} \rightarrow \Phi_{i-1 / 2} \widetilde{F}_{i-1 / 2}
$$

where

$$
\Phi_{i-1 / 2}= \begin{cases}\min \left(1, \Delta x\left(\mathbb{Q}_{i}^{\star}\right)^{1} / \Delta t \mathcal{M}_{i}\right) \quad \text { if } & \widetilde{F}_{i-1 / 2}^{1}<0, \\ \min \left(1, \Delta x\left(\mathbb{Q}_{i-1}^{\star}\right)^{1} / \Delta t \mathcal{M}_{i-1}\right) & \text { if } \quad \widetilde{F}_{i-1 / 2}^{1}>0 .\end{cases}
$$

\subsection{Determination of the Riemann structure}

In this section a summary of the procedure for determining the exact Riemann structure of the shallow-water equations is provided. Consider the homogeneous shallow-water equations

$$
\begin{aligned}
(h \sigma)_{t}+(h u \sigma)_{x} & =0, \\
(h u \sigma)_{t}+\left(h u^{2} \sigma+\frac{1}{2} g h^{2} \sigma\right)_{x} & =0,
\end{aligned}
$$

with

$$
\mathrm{q}(x, 0)=\left\{\begin{array}{ll}
\mathbf{q}_{l} & \text { if } \quad x<0, \\
\mathbf{q}_{r} & \text { if } \quad x>0,
\end{array} \quad \text { where } \quad \mathbf{q}=\left[\begin{array}{c}
h \sigma \\
h u \sigma
\end{array}\right] .\right.
$$

We would like to determine whether each characteristic field of (6.73) has a shock wave or a smooth rarefaction wave.

It can be shown that the integral curves of $r^{1}$ (q) of (6.73) have the functional form

$$
h u \sigma=h u^{\star} \sigma+2 h \sigma\left(\sqrt{g h^{\star}}-\sqrt{g h \frac{\sigma}{\sigma^{\star}}}\right),
$$

and the integral curves of $r^{2}(\mathrm{q})$ have the functional form

$$
h u \sigma=h u^{\star} \sigma-2 h \sigma\left(\sqrt{g h^{\star}}-\sqrt{g h \frac{\sigma}{\sigma^{\star}}}\right),
$$


for any fixed points $\left(h^{\star} \sigma^{\star}, u^{\star}\right)$ on the integral curves. In terms of the velocity instead of the momentum, we can rewrite (6.75) and (6.76) respectively as

$$
u=u^{\star}+2\left(\sqrt{g h^{\star}}-\sqrt{g h \frac{\sigma}{\sigma^{\star}}}\right),
$$

and

$$
u=u^{\star}-2\left(\sqrt{g h^{\star}}-\sqrt{g h \frac{\sigma}{\sigma^{\star}}}\right) .
$$

The expression (6.77) describes an integral curve of $r^{1}$, where $\left(h^{\star} \sigma^{\star}, u^{\star}\right)$ is an arbitrary point on the curve. This can be rewritten as

$$
u^{\star}+2 \sqrt{g h^{\star}}=u+2 \sqrt{g h \frac{\sigma}{\sigma^{\star}}} .
$$

Since $\left(h^{\star} \sigma^{\star}, u^{\star}\right)$ and $(h \sigma, u)$ are any two points on the curve, the function

$$
\mathrm{w}^{1}(\mathbf{q})=u+2 \sqrt{g h \frac{\sigma}{\sigma_{l}}},
$$

has the same value at all points on this curve. This is the Riemann invariant for the 1-family. Similarly, from (6.78) the Riemann invariant of the 2-family reads

$$
\mathrm{w}^{2}(\mathrm{q})=u-2 \sqrt{g h \frac{\sigma}{\sigma_{r}}} .
$$

Discontinuities must obey the Rankine-Hugoniot jump conditions [28, 22]. For the shallowwater equations (6.73) they take the form

$$
s\left[\left[\begin{array}{r}
h \sigma \\
h u \sigma
\end{array}\right]\right]=\left[\left[\begin{array}{r}
h u \sigma \\
h u^{2} \sigma+\frac{1}{2} g h^{2} \sigma
\end{array}\right]\right],
$$

where 【 \indicates the difference across the jump discontinuity and $s$ is the propagation speed of the discontinuity. The Rankine-Hugoniot conditions (6.81) can be used to derive the Hugoniot loci, curves in the phase space which connect two states through a shock wave. For the shallow-water equations (6.73), states $\mathrm{q}^{\star}$ that can be connected to $\mathrm{q}_{l}$ through a 1-shock satisfy

$$
u^{\star}=u_{l}+\sqrt{\frac{g}{2}\left(\frac{h_{l}}{h^{\star} \sigma^{\star}}-\frac{h^{\star}}{h_{l} \sigma_{l}}\right)\left(h_{l} \sigma_{l}-h^{\star} \sigma^{\star}\right)},
$$

and states $\mathrm{q}^{\star}$ that can be connected to $\mathrm{q}_{r}$ through a 2-shock satisfy

$$
u^{\star}=u_{r}-\sqrt{\frac{g}{2}\left(\frac{h_{r}}{h^{\star} \sigma^{\star}}-\frac{h^{\star}}{h_{r} \sigma_{r}}\right)\left(h_{r} \sigma_{r}-h^{\star} \sigma^{\star}\right)} .
$$

Definition 1. Lax entropy condition. A discontinuity separating states $\mathrm{q}_{l}$ and $\mathrm{q}_{r}$, propagating at speed s, satisfies the Lax entropy condition if there is an index $p$ such that

$$
\lambda^{p}\left(\mathbf{q}_{l}\right)>s>\lambda^{p}\left(\mathbf{q}_{r}\right)
$$


so that p characteristics are impinging on the discontinuity, while the other characteristics are crossing the discontinuity,

$$
\begin{aligned}
& \lambda^{j}\left(\mathbf{q}_{l}\right)<s \text { and } \lambda^{j}\left(\mathbf{q}_{r}\right)<s \text { for } j<p, \\
& \lambda^{j}\left(\mathbf{q}_{l}\right)>s \text { and } \lambda^{j}\left(\mathbf{q}_{r}\right)>s \text { for } j>p \text {, }
\end{aligned}
$$

where the eigenvalues are ordered so that $\lambda^{1}<\lambda^{2}<\cdots<\lambda^{m}$ in each state [11, 22].

Theorem 2. A solution to (6.73) is the correct entropy satisfying solution if and only if it has the following properties

1. A 1-shock connects $\mathrm{q}_{l}$ to $\mathrm{q}^{\star}$ if and only if $h^{\star}>h_{l}$.

2. A 2-shock connects $\mathrm{q}_{r}$ to $\mathrm{q}^{\star}$ if and only if $h^{\star}>h_{r}$.

3. Otherwise rarefactions defined by smooth integral curves of the first or second characteristic field connect $\mathrm{q}^{\star}$ to $\mathrm{q}_{l}$ or $\mathrm{q}_{r}$ respectively.

Proof of Theorem 2. Theorem 2 is the theorem 4.1 in [11] but with a slightly different proof here for the the shallow-water equations over variable cross-section. Suppose that a 2-wave connecting $\mathrm{q}_{r}$ to $\mathrm{q}^{\star}$ is a rarefaction. By (6.80) and the Lax entropy condition

$$
\begin{aligned}
0 & \leq \lambda^{2}\left(\mathbf{q}_{r}\right)-\lambda^{2}\left(\mathbf{q}^{\star}\right), \\
& =u_{r}+\sqrt{g h_{r}}-\left(u^{\star}+\sqrt{g h^{\star}}\right), \\
& =\mathrm{w}^{2}\left(\mathbf{q}_{r}\right)+3 \sqrt{g h_{r}}-\left(\mathrm{w}^{2}\left(\mathbf{q}^{\star}\right)+2 \sqrt{g h^{\star} \frac{\sigma^{\star}}{\sigma_{r}}}+\sqrt{g h^{\star}}\right), \\
& =3 \sqrt{g h_{r}}-\left(2 \sqrt{g h^{\star} \frac{\sigma^{\star}}{\sigma_{r}}}+\sqrt{g h^{\star}}\right),
\end{aligned}
$$

and hence

$$
9 h_{r} \geq h^{\star}\left(4 \frac{\sigma^{\star}}{\sigma_{r}}+1+4 \sqrt{\frac{\sigma^{\star}}{\sigma_{r}}}\right) .
$$

Now in order to satisfy $h^{\star} \leq h_{r}$ for any point $\left(h_{r} \sigma_{r}, u_{r}\right)$ on the integral curve of $r^{2}(\mathrm{q})$, we impose the following geometric constraint on the middle state cross-section $\sigma^{\star}$,

$$
\sigma^{\star} \geq \sigma_{r}
$$

Conversely, suppose that $h^{\star} \leq h_{r}$. If a 2-shock connects $\mathrm{q}^{\star}$ to $\mathrm{q}_{r}$, then the Lax entropy condition implies

$$
u^{\star}+\sqrt{g h^{\star}}>u_{r}+\sqrt{g h_{r}} \quad \Rightarrow \quad u^{\star}-u_{r}>\sqrt{g h_{r}}-\sqrt{g h^{\star}} \geq 0 \quad \text { (by supposition). }
$$

Additionally, $\mathrm{q}^{\star}$ and $\mathrm{q}_{r}$ must lie on the Hugoniot loci for a 2-shock, implying that

$$
u^{\star}-u_{r}=-\sqrt{\frac{g}{2}\left(\frac{h_{r}}{h^{\star} \sigma^{\star}}-\frac{h^{\star}}{h_{r} \sigma_{r}}\right)\left(h_{r} \sigma_{r}-h^{\star} \sigma^{\star}\right)} \leq 0,
$$


if $\sqrt{(\quad)} \geq 0$ in (6.88). However (6.87) and (6.88) are contradictory, and so if $h^{\star} \leq h_{r}$ then a 2 -rarefaction must connect $\mathrm{q}^{\star}$ to $\mathrm{q}_{r}$. Suppose that a 1-wave connecting $\mathrm{q}_{l}$ to $\mathrm{q}^{\star}$ is a rarefaction. By (6.79) and the Lax entropy condition

$$
\begin{aligned}
0 & \leq \lambda^{1}\left(\mathbf{q}^{\star}\right)-\lambda^{1}\left(\mathbf{q}_{l}\right) \\
& =u^{\star}-\sqrt{g h^{\star}}-\left(u_{l}-\sqrt{g h_{l}}\right), \\
& =\mathrm{w}^{1}\left(\mathbf{q}^{\star}\right)-2 \sqrt{g h^{\star} \frac{\sigma^{\star}}{\sigma_{l}}}-\sqrt{g h^{\star}}-\left(\mathrm{w}^{1}\left(\mathbf{q}_{l}\right)-3 \sqrt{g h_{l}}\right), \\
& =3 \sqrt{g h_{l}}-\left(2 \sqrt{g h^{\star} \frac{\sigma^{\star}}{\sigma_{l}}}+\sqrt{g h^{\star}}\right),
\end{aligned}
$$

and hence

$$
9 h_{l} \geq h^{\star}\left(4 \frac{\sigma^{\star}}{\sigma_{l}}+1+4 \sqrt{\frac{\sigma^{\star}}{\sigma_{l}}}\right) .
$$

And so in order to satisfy $h^{\star} \leq h_{l}$ for any point $\left(h_{l} \sigma_{l}, u_{l}\right)$ on the integral curve of $r^{1}(q)$, we impose the following geometric constraint on the middle state cross-section $\sigma^{\star}$,

$$
\sigma^{\star} \geq \sigma_{l}
$$

Conversely, suppose that $h^{\star} \leq h_{l}$. If a 1-shock connects $\mathrm{q}^{\star}$ to $\mathrm{q}_{l}$, then the Lax entropy condition implies

$$
u_{l}-\sqrt{g h_{l}}>u^{\star}-\sqrt{g h^{\star}} \quad \Rightarrow \quad u^{\star}-u_{l}<\sqrt{g h^{\star}}-\sqrt{g h_{l}} \leq 0 \quad \text { (by supposition). }
$$

Additionally, $\mathrm{q}^{\star}$ and $\mathrm{q}_{r}$ must lie on the Hugoniot loci for a 1-shock, implying that

$$
u^{\star}-u_{l}=\sqrt{\frac{g}{2}\left(\frac{h_{l}}{h^{\star} \sigma^{\star}}-\frac{h^{\star}}{h_{l} \sigma_{l}}\right)\left(h_{l} \sigma_{l}-h^{\star} \sigma^{\star}\right)} \geq 0,
$$

if $\sqrt{(\quad)} \geq 0$ in (6.92). However (6.91) and (6.92) are contradictory, and so if $h^{\star} \leq h_{l}$ then a 1-rarefaction must connect $\mathrm{q}^{\star}$ to $\mathrm{q}_{l}$. In summary, we set

$$
\sigma^{\star}=\max \left(\sigma_{l}, \sigma_{r}\right)
$$

in determination of the Riemann structure of the shallow-water equations (6.73).

Theorem 2 allows us to uniquely determine the middle state solution $\mathrm{q}^{\star}$ by using (6.79), (6.80), (6.82) and (6.83) such that the properties $1-3$ are satisfied. Defining the function

$$
\Phi^{1}\left(h, h_{l}\right)=\left\{\begin{array}{l}
2\left(\sqrt{g h \frac{\sigma}{\sigma_{l}}}-\sqrt{g h_{l}}\right) \quad \text { if } \quad h \leq h_{l}, \\
-\sqrt{\frac{g}{2}\left(\frac{h_{l}}{h \sigma}-\frac{h}{h_{l} \sigma_{l}}\right)\left(h_{l} \sigma_{l}-h \sigma\right)} \quad \text { if } \quad h>h_{l},
\end{array}\right.
$$

then we have

$$
u(h)=u_{l}-\Phi^{1}\left(h, h_{l}\right)
$$


for $u$ and $h$ connected through any 1 -wave to $u_{l}$. Similarly, defining the function

$$
\Phi^{2}\left(h, h_{r}\right)=\left\{\begin{array}{l}
2\left(\sqrt{g h \frac{\sigma}{\sigma_{r}}}-\sqrt{g h_{r}}\right) \quad \text { if } \quad h \leq h_{r}, \\
-\sqrt{\frac{g}{2}\left(\frac{h_{r}}{h \sigma}-\frac{h}{h_{r} \sigma_{r}}\right)\left(h_{r} \sigma_{r}-h \sigma\right)} \quad \text { if } h>h_{r},
\end{array}\right.
$$

then we have

$$
u(h)=u_{r}+\Phi^{2}\left(h, h_{r}\right),
$$

for $u$ and $h$ connected through any 2-wave to $u_{r}$. The middle state occurs in the phase space where the curves (6.94) and (6.96) intersect [11]. Subtracting (6.94) from (6.96) gives

$$
f(h)=\Delta u+\Phi^{1}\left(h, h_{l}\right)+\Phi^{2}\left(h, h_{r}\right), \quad h>0 .
$$

A root of $f(h)$ determines an intersection in phase space,

$$
f\left(h^{\star}\right)=\Delta u+\Phi^{1}\left(h^{\star}, h_{l}\right)+\Phi^{2}\left(h^{\star}, h_{r}\right)=0,
$$

which corresponds to a middle state depth $h^{\star}$. Since the function $f(h)$ is convex and monotonically increasing function of $h$, the Riemann structure can be determined by establishing one of the following three possibilities $[11,12]$

Case 1: $f\left(h_{\min }\right) \geq 0 \quad \Longleftrightarrow \quad$ two rarefactions ,

Case 2: $f\left(h_{\max }\right) \geq 0>f\left(h_{\min }\right) \Longleftrightarrow$ one shock, one rarefaction,

Case 3: $f\left(h_{\max }\right)<0 \quad \Longleftrightarrow \quad$ two shocks ,

where $h_{\min }=\min \left(h_{l}, h_{r}\right)$ and $h_{\max }=\max \left(h_{l}, h_{r}\right)$. In the event of Case $2, h_{l}<h_{r}$ implies a shock in the first family, and $h_{r}<h_{l}$ implies a shock in the second family.

\subsection{Riemann problems with transonic rarefactions}

As is discussed in [12] for problems with a large or transonic rarefaction in one field, an alternative definition of the pair $\left\{w_{i-1 / 2}^{2}, s_{i-1 / 2}^{2}\right\}$ allows a more accurate approximate Riemann solution. Given a large rarefaction in the first field, the pairs $\left\{w_{i-1 / 2}^{1}, s_{i-1 / 2}^{1}\right\}$ and $\left\{w_{i-1 / 2}^{2}, s_{i-1 / 2}^{2}\right\}$ can be used to approximate $\left\{r^{1}(\widetilde{\mathbf{q}}), \lambda^{1}(\widetilde{\mathrm{q}})\right\}$ at the left and right edges of the rarefaction. Similarly, given a large rarefaction in the second field, the pairs $\left\{w_{i-1 / 2}^{2}, s_{i-1 / 2}^{2}\right\}$ and $\left\{w_{i-1 / 2}^{3}, s_{i-1 / 2}^{3}\right\}$ can be used to approximate $\left\{r^{3}(\widetilde{\mathbf{q}}), \lambda^{3}(\widetilde{\mathbf{q}})\right\}$ at the left and right edges of the rarefaction. Since $r^{ \pm}(q)$ is only a function of its respective eigenvalues $\lambda^{ \pm}(q)$, in the event of a transonic 1-rarefaction an approximation to $\lambda^{-}\left(\mathbb{Q}_{i-1 / 2}^{*}\right)$ is only required to build the pair $\left\{w_{i-1 / 2}^{2}, s_{i-1 / 2}^{2}\right\}$. Using the Riemann invariant of the 1-family

$$
\lambda^{-}\left(\mathbb{Q}_{i-1 / 2}^{\star}\right)=\left(1+\frac{1}{2} \sqrt{\frac{\sigma_{l}}{\sigma^{\star}}}\right) u_{i-1 / 2}^{\star}-\frac{1}{2} \sqrt{\frac{\sigma_{l}}{\sigma^{\star}}}\left(u_{l}+2 \sqrt{g h_{l}}\right) .
$$


Similarly, in the event of a transonic 2-rarefaction an approximation to $\lambda^{+}\left(\mathbb{Q}_{i-1 / 2}^{\star}\right)$ is required to build the pair $\left\{w_{i-1 / 2}^{2}, s_{i-1 / 2}^{2}\right\}$. Using the Riemann invariant of the 2-family

$$
\lambda^{+}\left(\mathbb{Q}_{i-1 / 2}^{\star}\right)=\left(1+\frac{1}{2} \sqrt{\frac{\sigma_{r}}{\sigma^{\star}}}\right) u_{i-1 / 2}^{\star}-\frac{1}{2} \sqrt{\frac{\sigma_{r}}{\sigma^{\star}}}\left(u_{r}-2 \sqrt{g h_{r}}\right) .
$$

And hence an approximation to the middle state velocity $u_{i-1 / 2}^{\star}$ is required which is accomplished in $\$ 6.4$.

For the Riemann problem (6.73) the following speeds are defined as functions of $u_{i-1 / 2}^{\star}$, where it is the exact or just an approximation to the middle state velocity

$$
\begin{aligned}
& \lambda_{i-1 / 2}^{-\star}=\left(1+\frac{1}{2} \sqrt{\frac{\sigma_{l}}{\sigma^{\star}}}\right) u_{i-1 / 2}^{\star}-\frac{1}{2} \sqrt{\frac{\sigma_{l}}{\sigma^{\star}}}\left(u_{l}+2 \sqrt{g h_{l}}\right), \\
& \lambda_{i-1 / 2}^{+\star}=\left(1+\frac{1}{2} \sqrt{\frac{\sigma_{r}}{\sigma^{\star}}}\right) u_{i-1 / 2}^{\star}-\frac{1}{2} \sqrt{\frac{\sigma_{r}}{\sigma^{\star}}}\left(u_{r}-2 \sqrt{g h_{r}}\right) .
\end{aligned}
$$

Now in the event of a transonic 1-rarefaction we set

$$
\left\{w_{i-1 / 2}^{2}, s_{i-1 / 2}^{2}\right\}=\left\{\left(1, \lambda_{i-1 / 2}^{-\star}\left(u_{i-1 / 2}^{\star}\right),\left(\lambda_{i-1 / 2}^{-\star}\left(u_{i-1 / 2}^{\star}\right)\right)^{2}, 0,0\right)^{T}, \lambda_{i-1 / 2}^{-\star}\left(u_{i-1 / 2}^{\star}\right)\right\},
$$

and in the event of a transonic 2-rarefaction we set

$$
\left\{w_{i-1 / 2}^{2}, s_{i-1 / 2}^{2}\right\}=\left\{\left(1, \lambda_{i-1 / 2}^{+\star}\left(u_{i-1 / 2}^{\star}\right),\left(\lambda_{i-1 / 2}^{+\star}\left(u_{i-1 / 2}^{\star}\right)\right)^{2}, 0,0\right)^{T}, \lambda_{i-1 / 2}^{+\star}\left(u_{i-1 / 2}^{\star}\right)\right\} .
$$

In order to preserve depth positivity in the shallow regions where $h_{i-1 / 2}^{\star}$ approaches zero, the pair $\left\{w_{i-1 / 2}^{2}, s_{i-1 / 2}^{2}\right\}$ should be defined by the second of (6.48).

\subsection{Wet-dry fronts and the generalized Einfeldt speeds}

For the Riemann problems with an initial dry state to one side, the exact Riemann solution contains only a single rarefaction connecting the wet to the dry state $[12,29]$. In the event of a wet-dry front with an initially dry right state, the rarefaction is in the first characteristic field. In this case the speed of the wet-dry front can be determined using the Riemann invariant of the 1 -family which is

$$
-\frac{1}{2} \sqrt{\frac{\sigma_{l}}{\sigma^{\star}}}\left(u_{l}+2 \sqrt{g h_{l}}\right) .
$$

For an initially dry left state, the rarefaction is in the second characteristic field and the wet-dry interface propagates at a speed

$$
-\frac{1}{2} \sqrt{\frac{\sigma_{r}}{\sigma^{\star}}}\left(u_{r}-2 \sqrt{g h_{r}}\right)
$$

The speeds (6.106) and (6.107) correspond to (6.103) where the middle state velocity $u_{i-1 / 2}^{\star}$ corresponds to the initially dry state and is set to zero. 
For dry state problems the pair $\left\{w_{i-1 / 2}^{1}, s_{i-1 / 2}^{1}\right\}$ can still be interpreted as an approximation to the first field and the pair $\left\{w_{i-1 / 2}^{3}, s_{i-1 / 2}^{3}\right\}$ can be interpreted as an approximation to the second field of the shallow-water equations. This can be accomplished by adjusting the Einfeldt speeds in (6.49) in the case of an initial dry state and then use the same definition for the eigenpairs [12]. When the right state is initially dry we set

$$
\begin{aligned}
& s_{\mathcal{E}_{i-1 / 2}}^{1}=\min \left(\lambda^{1}\left(\mathbb{Q}_{i-1}^{n}\right), \widehat{\lambda}_{i-1 / 2}^{1}\right)=\lambda^{1}\left(\mathbb{Q}_{i-1}^{n}\right)=u_{i-1}-\sqrt{g h_{i-1}}, \\
& s_{\mathcal{E}_{i-1 / 2}}^{3}=\lambda_{i-1 / 2}^{-\star}\left(u_{i-1 / 2}^{\star}\right)=-\frac{1}{2} \sqrt{\frac{\sigma_{l}}{\sigma^{\star}}}\left(u_{l}+2 \sqrt{g h_{l}}\right),
\end{aligned}
$$

and when the left state is initially dry we set

$$
\begin{aligned}
& s_{\mathcal{E}_{i-1 / 2}}^{1}=\lambda_{i-1 / 2}^{+\star}\left(u_{i-1 / 2}^{\star}\right)=-\frac{1}{2} \sqrt{\frac{\sigma_{r}}{\sigma^{\star}}}\left(u_{r}-2 \sqrt{g h_{r}}\right), \\
& s_{\mathcal{E}_{i-1 / 2}^{3}}^{3}=\max \left(\lambda^{3}\left(\mathbb{Q}_{i}^{n}\right), \hat{\lambda}_{i-1 / 2}^{3}\right)=\lambda^{3}\left(\mathbb{Q}_{i}^{n}\right)=u_{i}+\sqrt{g h_{i}} .
\end{aligned}
$$

See [12] the detailed discussion about how the source terms should be adjusted for dry state Riemann problems.

For exact Riemann problems, the waves in the first field are never greater in speed than the waves in the second field. See the detailed discussion in $[22,12]$. For dry state problems the wave in one field becomes as fast as the wave in the other field, and so we have set $s_{\mathcal{E}_{i-1 / 2}}^{1}=\lambda_{i-1 / 2}^{+\star}$ when the left state is dry, and $s_{\mathcal{E}_{i-1 / 2}}^{3}=\lambda_{i-1 / 2}^{-\star}$ when the right state is dry. However, for near dry state problems, it is possible that the speed estimates are such that $s_{\mathcal{E}_{i-1 / 2}}^{1}>\lambda_{i-1 / 2}^{+\star}$ when the left state is near dry, and $s_{\mathcal{E}_{i-1 / 2}}^{3}<\lambda_{i-1 / 2}^{-\star}$ when the right state is near dry. Therefore the Einfeldt speeds are redefined by

$$
\begin{aligned}
& s_{\mathcal{E}_{i-1 / 2}}^{1}=\min \left(\lambda^{1}\left(\mathbb{Q}_{i-1}^{n}\right), \widehat{\lambda}_{i-1 / 2}^{1}, \lambda_{i-1 / 2}^{+\star}\left(u_{i-1 / 2}^{\star}\right)\right), \\
& s_{\mathcal{E}_{i-1 / 2}}^{3}=\max \left(\lambda^{3}\left(\mathbb{Q}_{i}^{n}\right), \widehat{\lambda}_{i-1 / 2}^{3}, \lambda_{i-1 / 2}^{-\star}\left(u_{i-1 / 2}^{\star}\right)\right) .
\end{aligned}
$$

These speeds are the so-called generalized Einfeldt speeds. In this paper the eigenpairs are defined by (6.48) using the generalized Einfeldt speeds (6.110).

\subsection{Fractional step approach for the shallow-water equations with surge forcing function}

A fractional step approach can be used to solve the hyperbolic shallow water system (2.10) with a time-dependent surge forcing function. Shallow-water equations (2.10) can be augmented with a third equation for the flux of the momentum equation which is

$$
\left(h u^{2} \sigma+\frac{1}{2} g h^{2} \sigma\right)_{t}+\left(-u^{2}+g h\right)(h u \sigma)_{x}+2 u\left(h u^{2} \sigma+\frac{1}{2} g h^{2} \sigma\right)_{x}=2 u\left(\frac{1}{2} g h^{2} \sigma_{x}-g h \sigma \beta_{x}-\ddot{\xi} h \sigma\right) .
$$

After each time step of the augmented f-wave solver, a time step is taken in which the momentum equations are adjusted due to the horizontal acceleration of the vessel. For given $\mathbb{Q}_{i}^{n}$ apply 
the augmented f-wave solver (6.44) to update $\mathbb{Q}_{i}$ over time $\Delta t$ and call the new vector $\mathbb{Q}_{i}^{*}$. Then update $\mathbb{Q}_{i}^{\star}$ to $\mathbb{Q}_{i}^{n+1}$ by solving

$$
\left.\begin{array}{rl}
\frac{\partial}{\partial t}(h \sigma) & =0, \\
\frac{\partial}{\partial t}(h u \sigma) & =-\ddot{\xi} h \sigma, \\
\frac{\partial}{\partial t}\left(h u^{2} \sigma+\frac{1}{2} g h^{2} \sigma\right) & =-2 u \ddot{\xi} h \sigma .
\end{array}\right\}
$$

\section{Numerical results}

In this section we first validate the new augmented f-wave solver of $\S 6$, which is henceforth referred as $A 5 W S$ since it is based on equation (6.44), against the Roe-type solver of $\S 3$, which is hereafter referred as RoeTS, and then present some numerical results for the shallow water sloshing in an OWEL-type vessel with rigid-wall and open boundary conditions and with wetdry states. Then we validate the A5WS with a prescribed surge function using the fractional step approach of $\S 6.7$, which is hereafter referred as $F A 5 W S$, against the new Roe-type solver of $\S 4$, which is henceforth referred as NRoeTS, and then present some results for the shallow-water sloshing in a moving OWEL-type vessel.

\subsection{Convergence to steady state}

The first examples illustrate the long time convergence of transient solutions to a steady state for shallow water flow in a channel with vertical walls and a parabolic centered contraction over a hump. We present solutions for a subcritical flow, for a smooth transcritical flow, and for a non-smooth transcritical flow. The flow discharge $h u \sigma$ is imposed at inflow, and the fluid layer depth $h$ is imposed at outflow. The bottom topography is $\beta(x)=\max \left(.05\left(4-x^{2}\right), 0\right)$ and the cross-section contraction is described by a quadratic interpolant. For the flows considered here the solution domain is $x \in[-10,10]$.

Figure 2 shows convergence to steady state for a subcritical flow. It is borrowed from [17] (see Figure 5 in [17]). The initial conditions and input parameters are

$$
(h u \sigma)_{\text {in }}=4.42, \quad h_{\text {out }}=2, \quad(\Delta x)_{\text {RoeTS }}=.1, \quad(\Delta x)_{A 5 W S}=.2, \quad \Delta t=.01 .
$$

The flow will remain subcritical provided the channel contraction is not too severe $\left(\sigma_{\min }>\right.$ .8842) [4]. For our example here $\sigma_{\min }=.90$. The channel geometry and computed large time solutions, $h+\beta$, for the RoeTS and A5WS solvers are displayed in the first row. Numerical results are in excellent agreement. Smooth steady state solutions are characterized by two constants, the flow rate $h u \sigma=$ Const and the energy $E=\frac{1}{2} u^{2}+g(h+\beta)=$ Const, and so the steady state of rest is recognized as $u=0$ and $h(x)+\beta(x)=$ Const. The second and third rows in Figure 2 show the computed flow rate and energy at $t=120 \mathrm{~s}$ for the RoeTS and A5WS solvers, respectively. The closeness of the flow rate and energy to being constants shows how well the RoeTS and A5WS solvers approximate general steady state solutions. The 

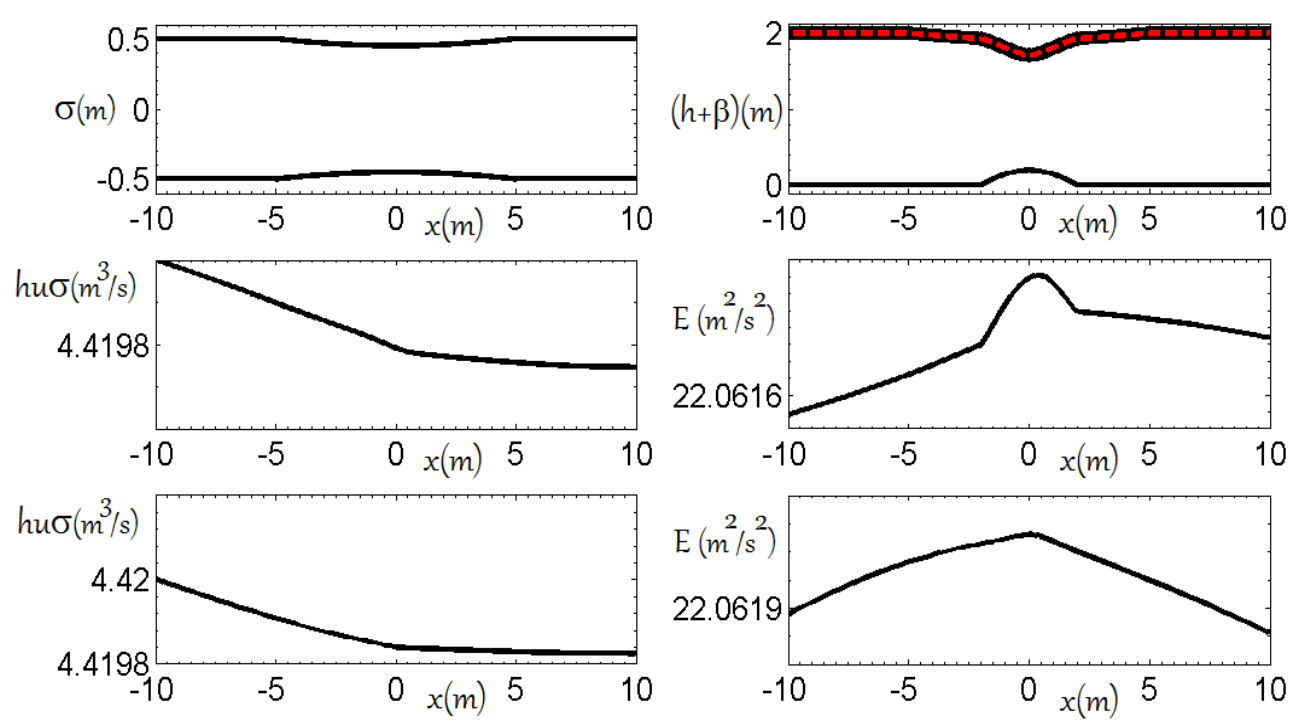

Figure 2: Comparison with Figure 5 in [17]. First row left: channel cross-section, horizontal axis is $x(m)$ and vertical axis is $\sigma(x)$. First row right: bottom topography $\beta(x)$ and water level $h(x)+\beta(x)$ at $t=120 \mathrm{~s}$ at steady state for subcritical flow. The numerical solution of the RoeTS is shown in solid black line and for the A5WS in dashed red line. Second row left: discharge $h u \sigma$ versus $x(m)$ at $t=120 \mathrm{~s}$ for RoeTS. Second row right: energy $E=$ $\frac{1}{2} u^{2}+g(h+\beta)$ versus $x(m)$ at $t=120 s$ for RoeTS. Third row left: discharge $h u \sigma$ versus $x(m)$ at $t=120 \mathrm{~s}$ for A5WS. Third row right: energy $E$ versus $x(m)$ at $t=120 \mathrm{~s}$ for A5WS.

$l_{\infty}$ error for $Q$ and $E$ for the RoeTS solver is $2.53 \times 10^{-4}$ and $5.11 \times 10^{-4}$, respectively. The $l_{\infty}$ error for $Q$ and $E$ for the A5WS solver is $1.74 \times 10^{-4}$ and $1.93 \times 10^{-4}$, respectively.

Figure 3 shows convergence to steady state for a smooth transcritical flow. The channel has narrower contraction than the one considered in the subcritical case $\left(\sigma_{\min }<.8842\right)$. For our example here $\sigma_{\min }=.70$. The initial conditions and input parameters are

$$
(h u \sigma)_{\text {in }}=1.53, \quad h_{\text {out }}=.3384, \quad(\Delta x)_{\text {RoeTS }}=.1, \quad(\Delta x)_{A 5 W S}=.2, \quad \Delta t=.01 .
$$

The channel geometry and computed large time solutions, $h+\beta$, for the RoeTS and A5WS solvers are displayed in the first row. Numerical results are in excellent agreement with each other and also with Figure 5 in [17]. The second and third rows in Figure 3 show that the both solvers are able to maintain the smooth steady state without spurious oscillations. By design, both solvers respect general steady state solutions. The closeness of the flow rate and energy to being constants shows how well the RoeTS and A5WS solvers approximate general steady state solutions. The jump in $Q$ and $E$ at outflow is because of the imposed boundary condition for the fluid layer depth $h_{\text {out }}$ and how the value of $u_{\text {out }}$ is computed in the code. The computed solutions for the A5WS are with coarse grid using 100 grid points.

Figure 4 shows convergence to steady state for a transcritical flow with a jump. The channel has narrower contraction such that $\sigma_{\text {min }}=.66$. The steady state solution corresponds to a flow accelerating from subcritical to supercritical as it turns over the hump and through the contraction, then decelerates abruptly to subcritical flow through a hydraulic jump in order to 

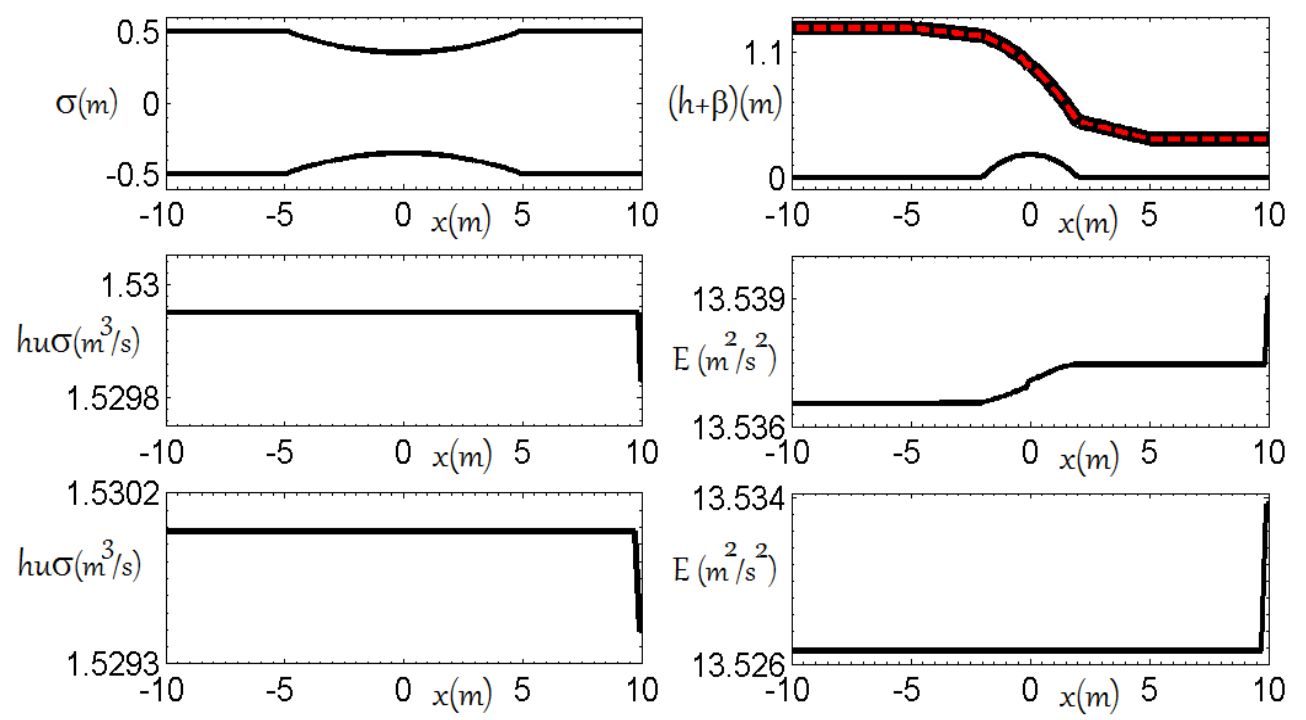

Figure 3: Comparison with Figure 5 in [17]. First row left: channel cross-section, horizontal axis is $x(m)$ and vertical axis is $\sigma(x)$. First row right: bottom topography $\beta(x)$ and water level $h(x)+\beta(x)$ at $t=120 \mathrm{~s}$ at steady state for smooth transcritical flow. The numerical solution of the RoeTS is shown in solid black line and for the A5WS in dashed red line. Second row left: discharge $h u \sigma$ versus $x(m)$ at $t=120 \mathrm{~s}$ for RoeTS. Second row right: energy $E=$ $\frac{1}{2} u^{2}+g(h+\beta)$ versus $x(m)$ at $t=120 s$ for RoeTS. Third row left: discharge $h u \sigma$ versus $x(m)$ at $t=120 s$ for A5WS. Third row right: energy $E$ versus $x(m)$ at $t=120 s$ for A5WS.

match the outflow boundary condition [4]. The initial conditions and input parameters are

$$
(h u \sigma)_{\text {in }}=.18, \quad h_{\text {out }}=.34, \quad(\Delta x)_{\text {RoeTS }}=.1, \quad(\Delta x)_{A 5 W S}=.2, \quad \Delta t=.01 .
$$

The channel geometry and computed large time wave profile for the RoeTS and A5WS solvers are shown in the first row. Numerical results are in excellent agreement with each other and also with Figure 5 in [17]. The second and third rows in Figure 4 show the computed flow rate and energy at $t=120 \mathrm{~s}$ for the RoeTS and A5WS solvers, respectively. The closeness of $Q$ and $E$ to being (piecewise) constants is a good measure of how well the solvers do in approximating general steady states. A single cell spike in $Q$ and an overshoot in $E$ are due the boundary conditions $Q_{i n}$ and $h_{\text {out }}$ and the narrow channel contraction which cause subcriticalsupercritical-subcritical transition in fluid flow over the hump and through the contraction. Similar computations are shown in Figure 11 of [4] for different boundary conditions and channel contraction.

\subsection{Sloshing in an OWEL-type vessel with wet-dry states}

In this section some simulations are presented for shallow water sloshing inside an OWEL type vessel with rigid wall and inflow-outflow boundary conditions. This is motivated by modelling shallow fluid flows in the ocean WEC proposed by Offshore Wave Energy Ltd, a schematic of which can be found on the website [1]. OWEL is a floating rectangular device, open at one end to allow waves in. Once they are trapped, the waves undergo interior fluid sloshing. A rise in 

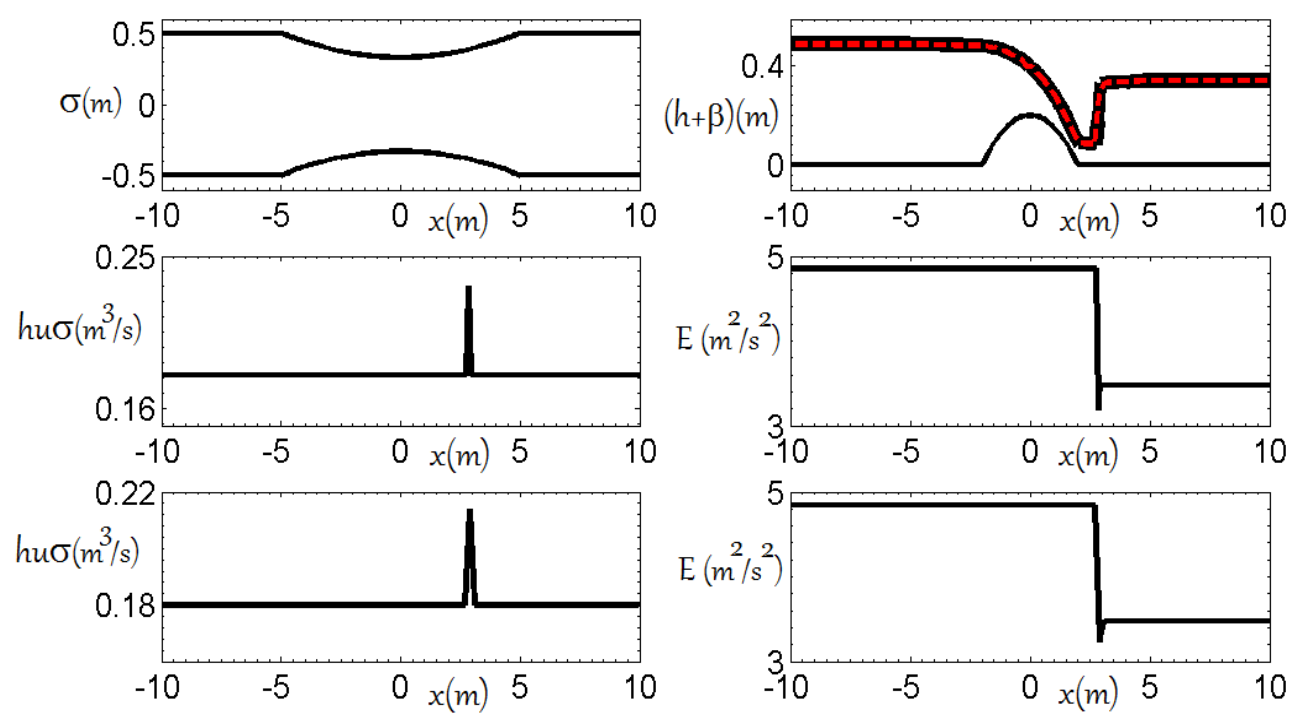

Figure 4: Comparison with Figure 5 in [17]. First row left: channel cross-section, horizontal axis is $x(m)$ and vertical axis is $\sigma(x)$. First row right: bottom topography $\beta(x)$ and water level $h(x)+\beta(x)$ at $t=120 s$ at steady state for transcritical flow with a jump. The numerical solution of the RoeTS is shown in solid black line and for the A5WS in dashed red line. Second row left: discharge $h u \sigma$ versus $x(m)$ at $t=120 \mathrm{~s}$ for RoeTS. Second row right: energy $E=$ $\frac{1}{2} u^{2}+g(h+\beta)$ versus $x(m)$ at $t=120 s$ for RoeTS. Third row left: discharge $h u \sigma$ versus $x(m)$ at $t=120 \mathrm{~s}$ for A5WS. Third row right: energy $E$ versus $x(m)$ at $t=120 \mathrm{~s}$ for A5WS.

the wave height is induced within the duct, mainly due to the internal geometry of the WEC. The wave then creates a seal with the rigid lid resulting in a moving trapped pocket of air ahead of the wave front which drives the power take off. It is anticipated that the first commercial platform of the OWEL WEC would have an overall length of $70 \mathrm{~m}$, inlet width of $22 \mathrm{~m}$, and draft of $8 m$ with rated power of $2 M W$ [1].

For the first simulation the bottom profile is set to be

$$
\beta(x)= \begin{cases}\beta_{0} \text { for } 0<x \leq x_{0}, \\ \beta_{0}+\frac{\beta\left(x_{1}\right)-\beta_{0}}{x_{1}-x_{0}}\left(x-x_{0}\right) & \text { for } \quad x_{0}<x \leq x_{1}, \\ \beta\left(x_{1}\right)-\frac{\beta\left(x_{1}\right)}{L-x_{1}}\left(x-x_{1}\right) & \text { for } \quad x_{1}<x \leq L,\end{cases}
$$

and the cross-section is set to be

$$
\sigma(x)=\left\{\begin{array}{l}
\sigma_{0} \text { for } 0 \leq x \leq x_{0}, \\
\sigma_{0}+2 s_{0}\left(x_{1}-x_{0}\right) \quad \text { for } \quad x_{0}<x \leq x_{1} \\
\sigma\left(x_{1}\right)+2 s_{1}\left(x-x_{1}\right) \quad \text { for } \quad x_{1}<x \leq L .
\end{array}\right.
$$

The initial conditions are

$$
u(x, 0)=0 \text { for } 0 \leq x \leq L
$$



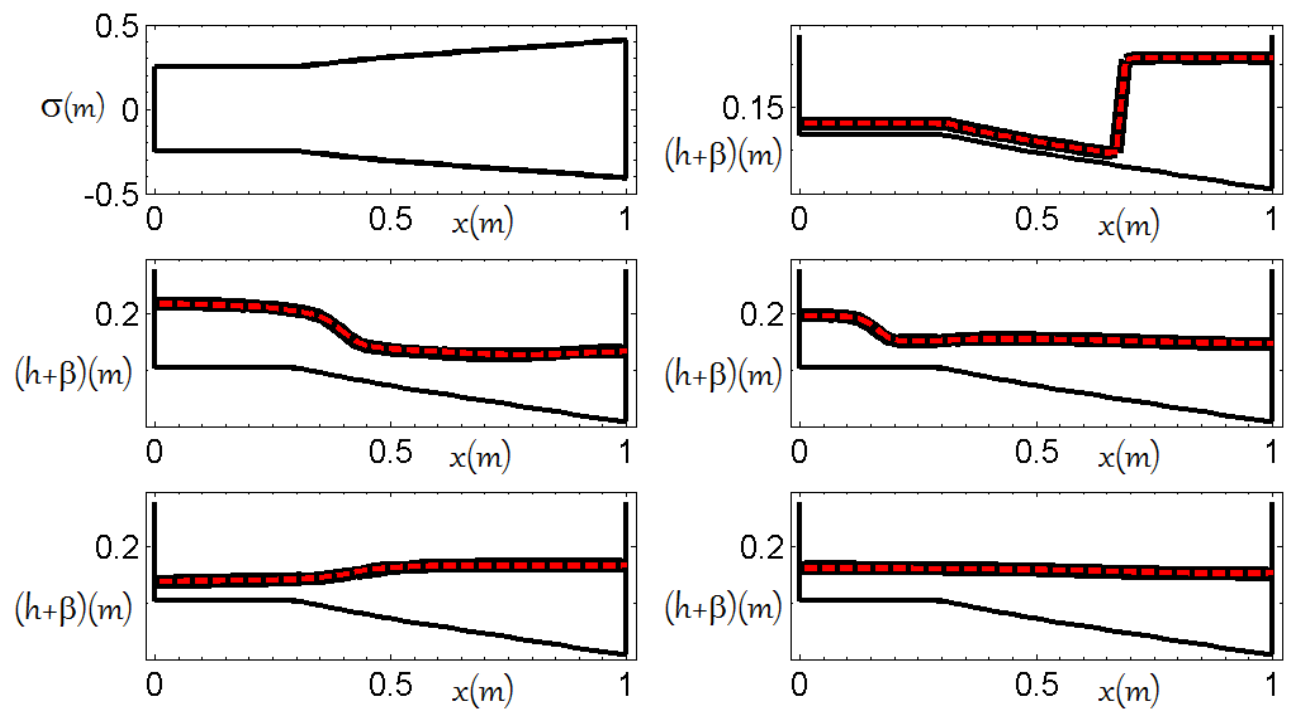

Figure 5: A5WS solver versus RoeTS solver. First row from left to right: vessel cross-section versus $x(m)$ and snapshot of the wave profile at $t=.001 \mathrm{~s}$. Second row from left to right: snapshots of the wave profile at $t=1 \mathrm{~s}$ and $t=2.7 \mathrm{~s}$. Third row from left to right: snapshots of the wave profile at $t=4.1 \mathrm{~s}$ and $t=10 \mathrm{~s}$. Computed solutions are shown in solid line for the RoeTS and in dashed line for the A5WS.

and

$$
h(x, 0)=\left\{\begin{array}{l}
h_{0}-\beta(x) \text { for } 0 \leq x \leq x_{0}, \\
h_{1} \text { for } x_{0}<x \leq x_{1}, \\
h_{2} \text { for } x_{1}<x \leq x_{2}, \\
h_{3}-\beta(x) \text { for } x_{2} \leq x \leq L .
\end{array}\right.
$$

The boundary conditions are

$$
u(0, t)=u(L, t)=0
$$

Take the input parameters as

$$
\begin{aligned}
L & =1 \mathrm{~m}, \quad \beta_{0}=.1 \mathrm{~m}, \quad x_{0}=L / 3, \quad h_{0}=.12 \mathrm{~m}, \quad h_{1}=.02 \mathrm{~m}, \quad h_{2}=.02 \mathrm{~m}, \\
\beta\left(x_{1}\right) & =.07 \mathrm{~m}, \quad h_{3}=.24 \mathrm{~m}, \quad x_{1}=L / 2, \quad x_{2}=2 L / 3, \quad \sigma_{0}=.5 \mathrm{~m}, \quad s_{0}=.3 \\
\Delta x & =.02 \mathrm{~m}, \quad \Delta t=.001 \mathrm{~s}, \quad s_{1}=.2
\end{aligned}
$$

In this example the RoeTS and A5WS solvers are validated against each other. Figure 5 depicts the cross-section of the vessel and the computed snapshots of the wave profile up to $10 \mathrm{~s}$ for both solvers. Numerical results are in excellent agreement.

For the next simulation the initial conditions and the input parameters are set to be the same as the previous simulation but with $h_{3}=.18 \mathrm{~m}$ and influx-efflux boundary conditions for the fluid velocity. Denoting the interior values of the cell velocities by $u_{1}^{n}, \ldots, u_{N}^{n}$ then the ghost cell values are set to be

$u_{0}^{n}=.1 \cos (2 n \Delta t), \quad u_{-1}^{n}=.12 \cos (3 n \Delta t), \quad u_{N+1}^{n}=-.14 \cos (3 n \Delta t), \quad u_{N+2}^{n}=-.1 \cos (2 n \Delta t)$. 

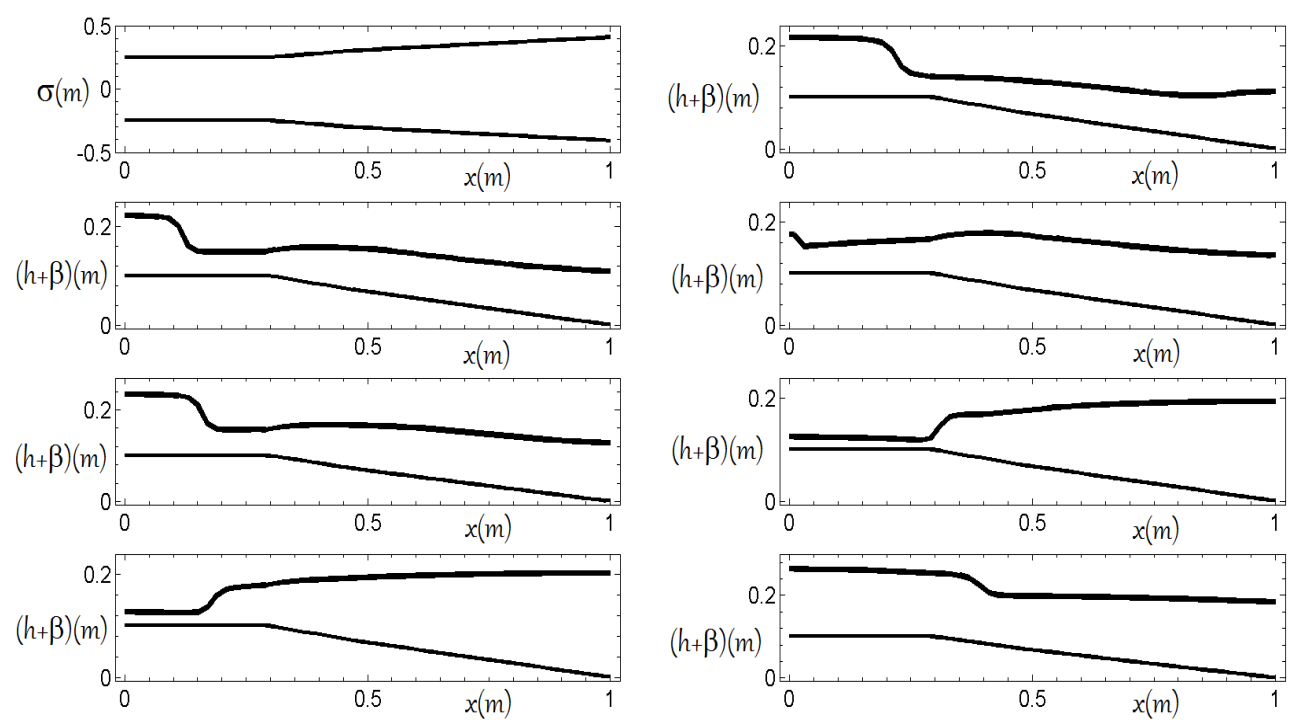

Figure 6: Shallow water flow inside an OWEL type vessel with influx-efflux boundary conditions. First row from left to right: vessel cross-section versus $x(m)$ and snapshot of the wave profile at $t=1 \mathrm{~s}$ with A5WS. Second row from left to right: snapshots of the wave profile at $t=2.8 \mathrm{~s}$ and $t=4.7 \mathrm{~s}$. Third row from left to right: snapshots of the wave profile at $t=9.1 \mathrm{~s}$ and $t=14.7 \mathrm{~s}$. Fourth row from left to right: snapshots of the wave profile at $t=21 \mathrm{~s}$ and $t=30 \mathrm{~s}$.

Figure 6 depicts the cross-section of the vessel and the computed snapshots of the wave profile up to $30 s$ for the A5WS with influx-efflux boundary conditions.

The next simulation is devoted to the propagation of wet-dry fronts inside an OWEL-type vessel with rigid-wall boundary conditions. The initial conditions are quiescent fluid with bottom topography, cross-section and initial wave height defined in (7.113), (7.114) and (7.116), respectively, with the input parameters

$$
\begin{aligned}
L & =1 \mathrm{~m}, \quad \beta_{0}=.1 \mathrm{~m}, \quad x_{0}=L / 3, \quad h_{0}=.1000001 \mathrm{~m}, \quad h_{1}=10^{-7} \mathrm{~m}, \quad h_{2}=10^{-7} \mathrm{~m}, \\
\beta\left(x_{1}\right) & =.07 \mathrm{~m}, \quad h_{3}=.20 \mathrm{~m}, \quad x_{1}=L / 2, \quad x_{2}=2 L / 3, \quad \sigma_{0}=.5 \mathrm{~m}, \quad s_{0}=.3, \\
\Delta x & =.02 \mathrm{~m}, \quad \Delta t=.001 \mathrm{~s}, \quad s_{1}=.2 .
\end{aligned}
$$

Figure 7 depicts the cross-section of the vessel and the snapshots of shallow-water sloshing with wet-dry fronts up to $5 s$ for the A5WS solver.

\subsection{Sloshing in an OWEL-type vessel with prescribed surge forcing}

In the next simulation we compare the shallow-water sloshing inside an OWEL-type vessel with prescribed surge forcing between the solvers FA5WS and NRoeTS. The boundary conditions are the no-flow boundary conditions. The initial conditions are quiescent fluid with bottom topography, cross-section and initial wave height defined in (7.113), (7.114) and (7.116), re- 

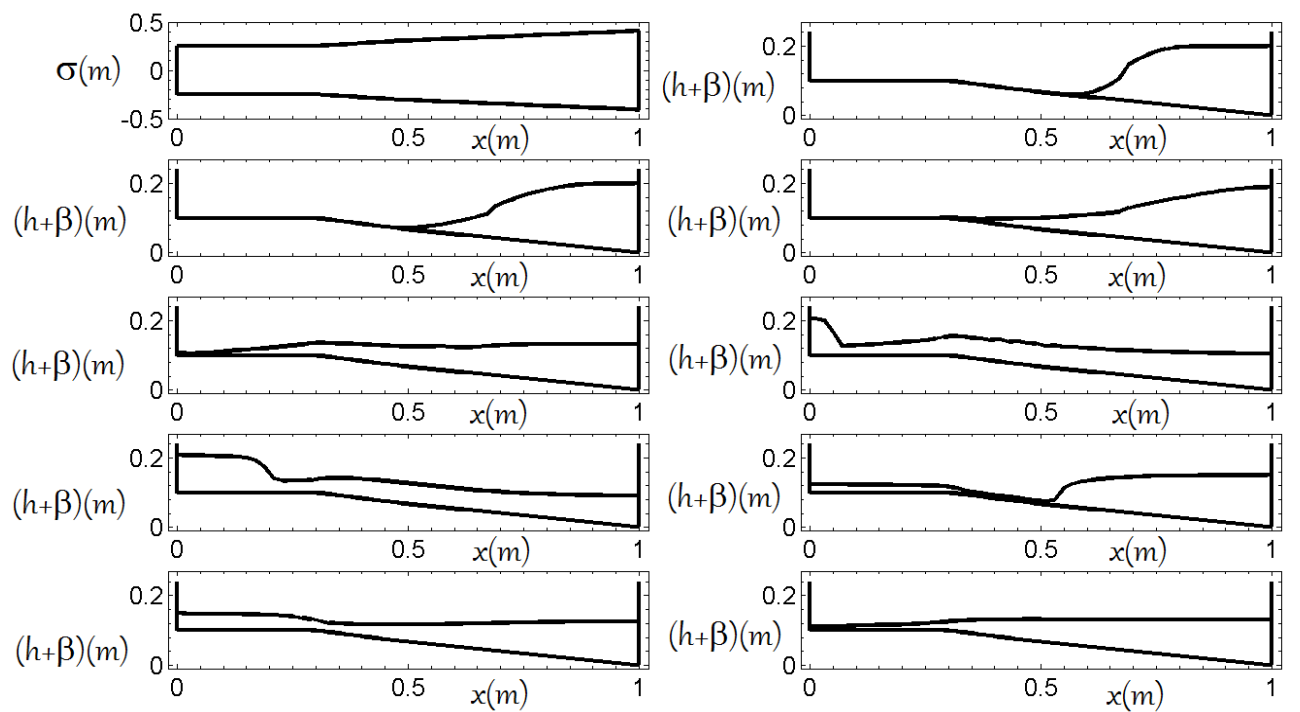

Figure 7: Wet-dry fronts: first row from left to right: vessel cross-section versus $x(m)$ and snapshot of the wave profile at $t=.05 \mathrm{~s}$ with A5WS. Second row from left to right: snapshots of the wave profile at $t=.1 \mathrm{~s}$ and $t=.2 \mathrm{~s}$. Third row from left to right: snapshots of the wave profile at $t=.4 \mathrm{~s}$ and $t=.6 \mathrm{~s}$. Fourth row from left to right: snapshots of the wave profile at $t=.9 \mathrm{~s}$ and $t=2.1 \mathrm{~s}$. Fifth row from left to right: snapshots of the wave profile at $t=3.4 \mathrm{~s}$ and $t=5 \mathrm{~s}$.

spectively, with the input parameters

$$
\begin{aligned}
L & =1 m, \quad \beta_{0}=.1 m, \quad x_{0}=L / 3, \quad h_{0}=.14 m, \quad h_{1}=.03 m, \quad h_{2}=.03 m \\
\beta\left(x_{1}\right) & =.07 m, \quad h_{3}=.24 m, \quad x_{1}=L / 2, \quad x_{2}=2 L / 3, \quad \sigma_{0}=.5 m, \quad s_{0}=.3, \\
\Delta x & =.02 m, \quad \Delta t=.001 s, \quad s_{1}=.2
\end{aligned}
$$

The surge forcing function is set to be

$$
\xi(t)=\varepsilon \sin (\omega t),
$$

with $\varepsilon=.02 \mathrm{~m}$ and $\omega=2.0451 \mathrm{rad} / \mathrm{s}$. Figure 8 depicts the cross-section of the vessel and the snapshots of shallow-water sloshing up to $8 s$ for the FA5WS and NRoeTS solvers. Numerical solutions are in good agreement. However increasing the angular frequency would result in phase error between the numerical solutions. The phase error can be a result of approximations for the middle state solutions in the NRoeTS solver for the entropy fix. Small discrepancies between the numerical solutions in Figure 8 are due to middle state approximations in the NRoeTS solver.

The next simulation is devoted to the shallow water sloshing inside an OWEL-type vessel under prescribed surge forcing near or with wet-dry states. The vessel geometry, boundary conditions 

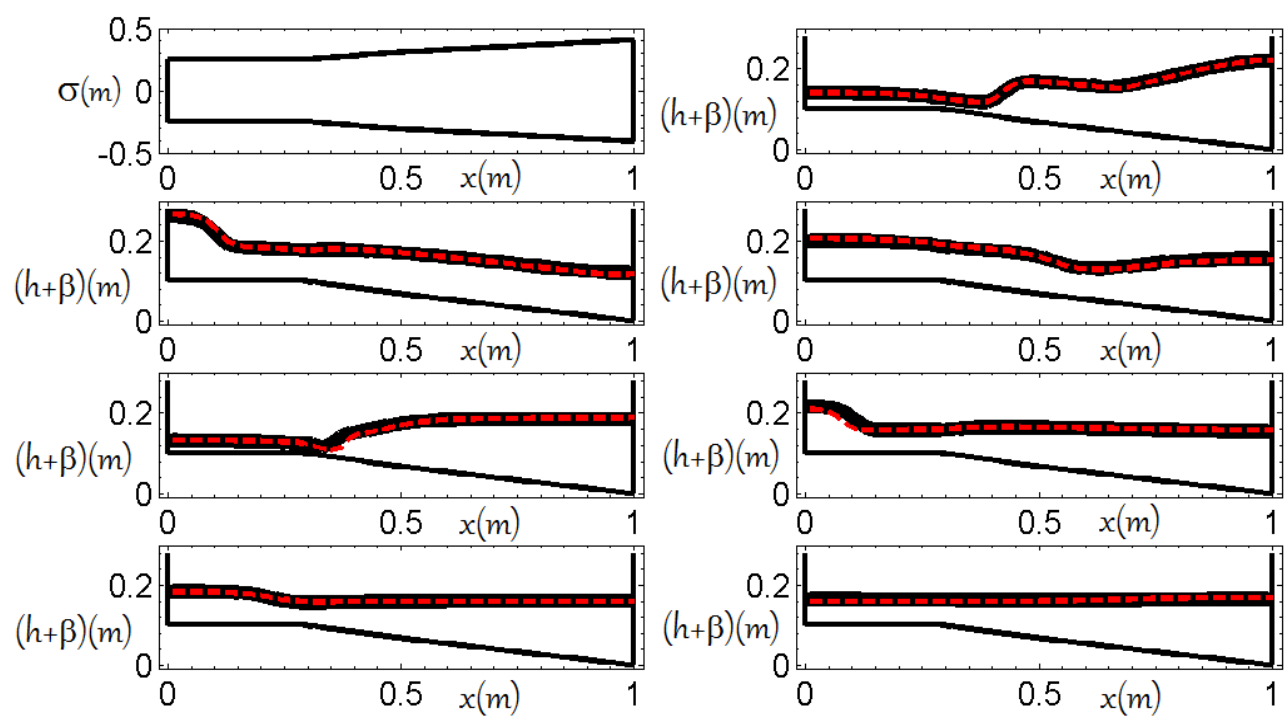

Figure 8: FA5WS solver versus NRoeTS solver. Sloshing inside an OWEL type vessel with prescribed surge forcing: first row from left to right: vessel cross-section versus $x(m)$ and snapshot of the wave profile at $t=.2 \mathrm{~s}$. Second row from left to right: snapshots of the wave profile at $t=.7 \mathrm{~s}$ and $t=1.1 \mathrm{~s}$. Third row from left to right: snapshots of the wave profile at $t=1.9 \mathrm{~s}$ and $t=2.5 \mathrm{~s}$. Fourth row from left to right: snapshots of the wave profile at $t=4.8 \mathrm{~s}$ and $t=8 \mathrm{~s}$. Computed solutions are shown in solid line for the FA5WS and in dashed red line for the NRoeTS.

and initial conditions are set the same as the previous simulation but with the input parameters

$$
\begin{aligned}
L & =1 m, \quad \beta_{0}=.1 m, \quad x_{0}=L / 3, \quad h_{0}=.21 m, \quad h_{1}=.04 m, \quad h_{2}=.04 m, \\
\beta\left(x_{1}\right) & =.07 m, \quad h_{3}-\beta(x)=.04 m, \quad x_{1}=L / 2, \quad x_{2}=2 L / 3, \quad \sigma_{0}=.5 m, \quad s_{0}=.3, \\
\Delta x & =.02 m, \quad \Delta t=.001 s, \quad s_{1}=.2
\end{aligned}
$$

and $\varepsilon=.03 \mathrm{~m}$ and $\omega=1.967 \mathrm{rad} / \mathrm{s}$. Figures 9 and 10 depict the cross-section of the vessel and the snapshots of the shallow-water sloshing near dry states up to $9 s$ for the FA5WS solver.

\section{Concluding remarks}

This paper is devoted to derivation of the shallow-water equations over variable cross-section and bottom topography under the surge acceleration. The motivation is modelling of shallowwater flows in ocean wave energy harvesters. The main goal of the paper is to develop wellbalanced finite volume solvers for these equations which could handle wet-dry states. For this purpose a new version of George's augmented f-wave solvers is derived. This approximate Riemann solver decomposes an augmented state variable, the wetted cross-section, momentum, momentum flux, bottom topography, and cross-section function into five waves. By defining the eigenspeeds and the eigenvectors appropriately, the augmented solver is able to maintain several nice features in the approximate solution which are preserving depth positivity with 

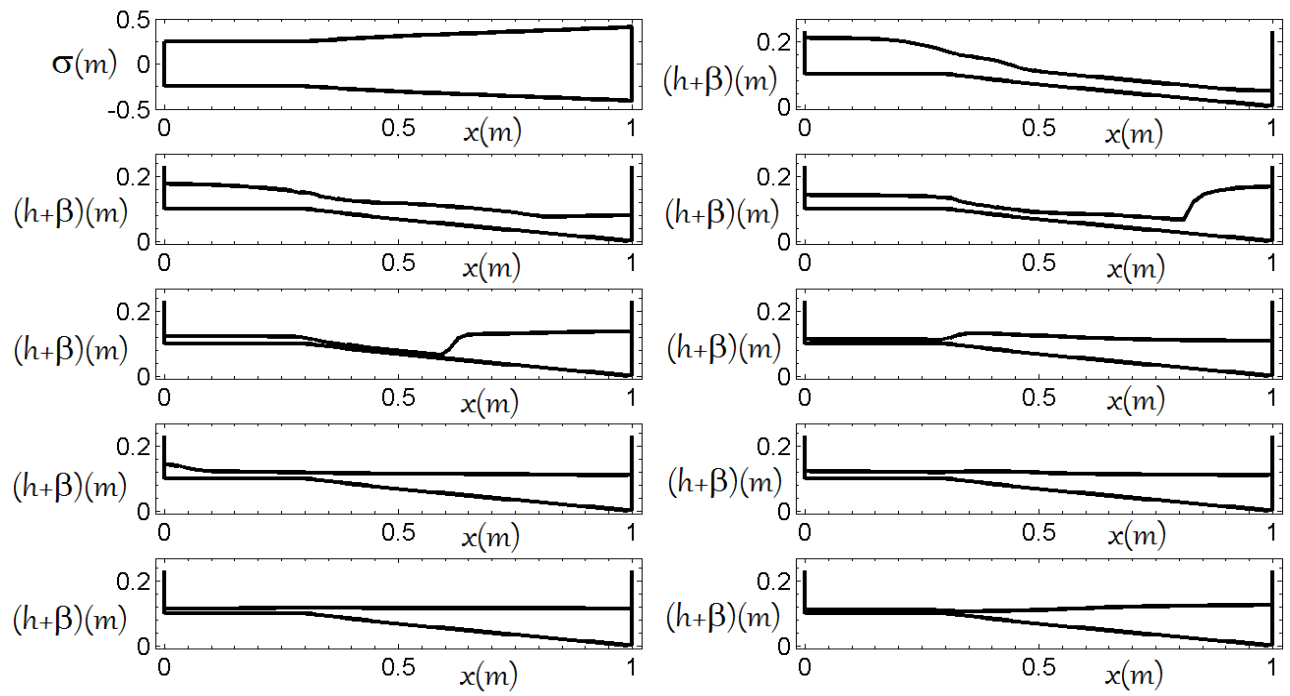

Figure 9: Sloshing inside an OWEL type vessel with prescribed surge forcing near dry states with FA5WS solver: first row from left to right: vessel cross-section versus $x(m)$ and snapshot of the wave profile at $t=.1 \mathrm{~s}$. Second row from left to right: snapshots of the wave profile at $t=.4 \mathrm{~s}$ and $t=.7 \mathrm{~s}$. Third row from left to right: snapshots of the wave profile at $t=1.1 \mathrm{~s}$ and $t=1.6 \mathrm{~s}$. Fourth row from left to right: snapshots of the wave profile at $t=2.2 \mathrm{~s}$ and $t=3 \mathrm{~s}$. Fifth row from left to right: snapshots of the wave profile at $t=3.5 \mathrm{~s}$ and $t=4 \mathrm{~s}$.
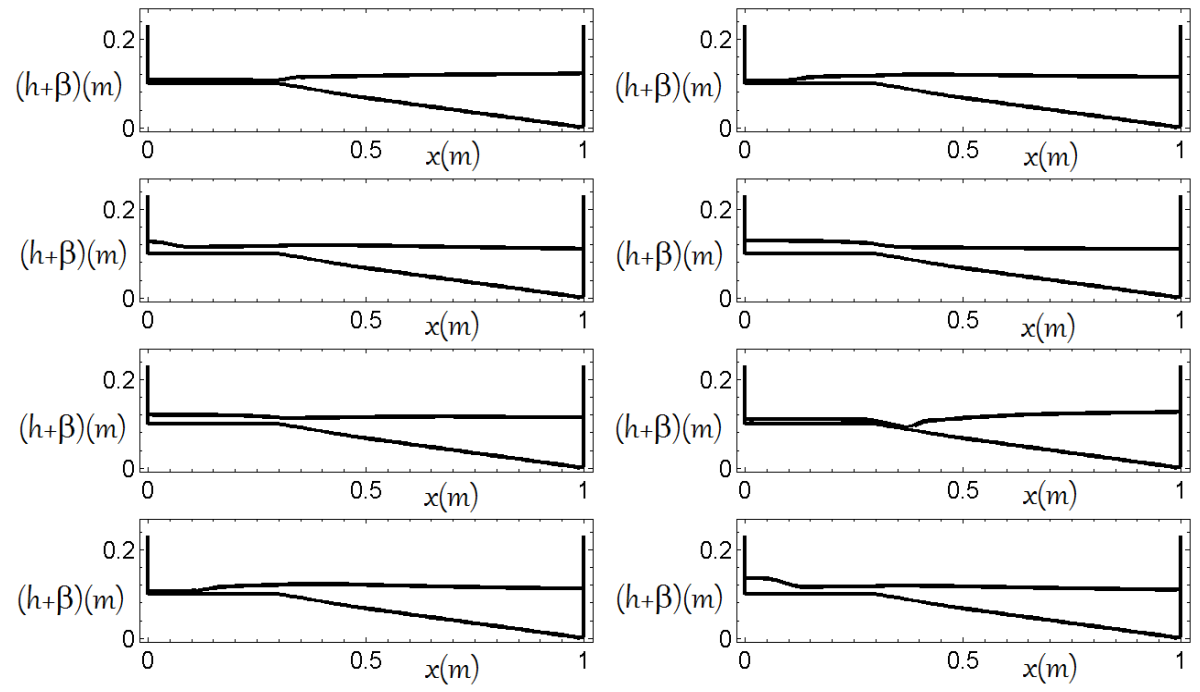

Figure 10: Continued: first row from left to right: snapshot of the wave profile at $t=4.6 \mathrm{~s}$ and $t=5.1 \mathrm{~s}$. Second row from left to right: snapshots of the wave profile at $t=5.5 \mathrm{~s}$ and $t=6.3 \mathrm{~s}$. Third row from left to right: snapshots of the wave profile at $t=7 \mathrm{~s}$ and $t=7.7 \mathrm{~s}$. Fourth row from left to right: snapshots of the wave profile at $t=8.5 \mathrm{~s}$ and $t=9 \mathrm{~s}$. 
the geometric source terms, providing an exact solution for a single shock Riemann problem since the solver is equivalent to the Roe solver for such Riemann problems, having a natural entropy fix for transonic rarefactions, and being well-balanced in the sense that the augmented solver preserves steady state solutions with the geometric source terms. This new augmented f-wave solver is validated against the Roe-type solver of [17], and a review of its derivation is given in this paper. Numerical simulations for wetting and drying of the bottom profile in an OWEL type Wave Energy Converter [1] are presented. Also numerical simulations with open boundary conditions are presented. A fractional step approach is used to deal with the shallowwater equations under prescribed surge forcing using the new augmented f-wave solvers. The numerical results of the time-dependent augmented f-wave solver are compared with a new Roe-type solver for the shallow-water equations over variable cross-section with horizontal acceleration which its derivation is given in the paper.

A direction of great interest is to modify the current new augmented f-wave solvers to include wetting and drying of a rigid-lid in one-layer shallow-water flows inside an OWEL type vessel with no-flow and open boundary conditions. This is motivated by modelling the power take off (PTO) system in the ocean wave energy converter proposed by Offshore Wave Energy Ltd. So the strategy going forward is to understand how the current augmented f-wave code could be modified to model a one-fluid wet-dry front at the rigid-lid.

Another feature of importance in OWEL WEC is the dynamic coupling between the vessel motion and the interior fluid motion. The current augmented f-wave solvers can be coupled to an ODE solver to study the dynamic coupling between the horizontal vessel motion and the interior one-layer shallow-water sloshing. A nonlinear model equation for the motion of an OWEL type vessel with interior shallow fluid sloshing can be derived from a variational principle. The equation is

$$
\frac{d}{d t}\left(\int_{0}^{L} \int_{\beta(x)}^{H(x)} \rho u(x, t) \sigma(x, z) d z d x+\left(m_{v}+m_{f}\right) \dot{\xi}\right)+\nu_{1} \xi-\nu_{2} \xi^{3}=0,
$$

where $m_{f}$ and $m_{v}$ are the mass of the fluid and the mass of the vessel, respectively, and $\nu_{1}>0$ and $\nu_{2}$ are constants. Of particular interest is the partition of energy between the vessel and fluid motion. So the main aim is to develop a dynamic coupling solver which respects the energy partition.

The presented augmented f-wave finite volume solvers, A5WS, might be applicable to other fields such as mathematical biology. For instance for simulation of modified shallow water equations as a mathematical model for blood flow in elastic arteries [14].

\section{Acknowledgments}

The research reported in this paper is supported by the EPSRC under Grant number EP/K008188/1. Due to confidentiality agreements with research collaborators, supporting data can only be made available to bona fide researchers subject to a non-disclosure agreement. Details of the data and how to request access are available from the University of Surrey publications repository: researchdata@surrey.ac.uk. The authors are grateful to both referees for their valuable comments. 


\section{References}

[1] http://www. owel.co.uk/

[2] H. Alemi Ardakani, T.J. Bridges \& M.R. Turner. Adaptation off-wave finite volume methods to the two-layer shallow-water equations in a moving vessel with a rigid-lid, Preprint (2014).

[3] H. Alemi Ardakani, T.J. Bridges \& M.R. Turner. Dynamic coupling between horizontal vessel motion and two-layer shallow-water sloshing, Preprint (2014).

[4] J. BALBÁS \& S. KARNI. A central scheme for shallow water flows along channels with irregular geometry, ESAIM: Mathematical Modelling and Numerical Analysis 43 333-351 (2009).

[5] D. Bale, R.J. LeVeque, S. Mitran \& J.A. Rossmanith. A wave propagation method for conservation laws and balance laws with spatially varying flux functions, SIAM J. Sci. Comput. 24 955-978 (2002).

[6] P. Brufau, M.E. VazQuez-Cendon \& P. Garcia-NaVArRo. A numerical model for the flooding and drying of irregular domains, Int. J. Numer. Meth. Fluids 39 247-275 (2002).

[7] M.J. Castro, J.A. Garcia-Rodriguez, J.M. Gonzalez-Vida, J. Macias, C. PARES \& M.E. VAZQUEZ-CENDON. Numerical simulation of two-layer shallow water flows through channels with irregular geometry, Journal of Computational Physics 195 202-235 (2004).

[8] B. EINFELDT. On Godunov-type methods for gas dynamics, SIAM J. Numer. Anal. 25 294-318 (1988).

[9] B. Einfeldt, C.D. Munz, P.L. Roe, \& B. Sjogreen. On Godunov-type methods for near low densities, Journal of Computational Physics 92 273-295 (1991).

[10] P. Garcia-Navarro \& M.E. VazQuez-Cendon. On numerical treatment of the source terms in the shallow water equations, Computers \& Fluids 29 951-979 (2000).

[11] D.L. GEORGE. Finite Volume Methods and Adaptive Refinement for Tsunami Propagation and Inundation, Ph.D. Thesis, University of Washington, Seattle, WA (2006).

[12] D.L. GEORGE. Augmented Riemann solvers for the shallow water equations over variable topography with steady states and inundation, Journal of Computational Physics 227 30893113 (2008).

[13] P. GLAISTER. Approximate Riemann solutions of the shallow water equations, Journal of Hydraulic Research 26 293-306 (1988).

[14] J.B. Grotberg \& O.E. Jensen. Biofluid mechanics in flexible tubes, Ann Rev Fluid Mech. 36 121-147 (2004).

[15] A. Harten \& J.M. Hyman. Self adjusting grid methods for one-dimensional hyperbolic conservation laws, Journal of Computational Physics 50 235-269 (1983). 
[16] A. Harten, P.D. LAX \& B. VAn LeER. On upstream differencing and Godunov-type schemes for hyperbolic conservation laws, SIAM Rev. 25 235-261 (1983).

[17] G. HernandeZ-Duenas \& S. KARni. Shallow water flows in channels, J. Sci. Comput. 48 190-208 (2011).

[18] J. KIM. Finite Volume Methods for Tsunami Generated by Submarine Landslides, Ph.D. Thesis, University of Washington, Seattle, WA (2014).

[19] J. Kim \& R.J. LeVEQUE. Two-layer shallow water system and its applications, Proceedings of 12th International Conference on Hyperbolic Problems, College Park, Maryland (2008).

[20] R.J. LEVEQUE. Wave propagation algorithms for multidimensional hyperbolic systems, Journal of Computational Physics 131 327-353 (1997).

[21] R.J. LEVEQUE. Balancing source terms and flux gradients in high-resolution Godunov methods: the quasi-steady wave-propagation algorithm, Journal of Computational Physics 146 346-365 (1998).

[22] R.J. LeVeque. Finite Volume Methods for Hyperbolic Problems, Cambridge Texts in Applied Mathematics, Cambridge University Press, Cambridge, UK (2002).

[23] R.J. LeVEQue \& D.L. George. High-Resolution Finite Volume Methods for the Shallow Water Equations with Bathymetry and Dry States, In Advanced Numerical Models for Simulating Tsunami Waves and Runup, P. L-F. Liu, H. Yeh \& C. Synolakis, editors, Advances in Coastal and Ocean Engineering, World Scientific $1043-73$ (2007).

[24] K.T. MANDLI. Finite Volume Methods for the Multilayer Shallow Water Equations with Applications to Storm Surge, Ph.D. Thesis, University of Washington, Seattle, WA (2011).

[25] K.T. MANDLI. A numerical method for the two layer shallow water equations with dry states, Ocean Modelling 72 80-91 (2013).

[26] P.L. RoE. Approximate Riemann solvers, parameter vectors, and difference schemes, Journal of Computational Physics 43 357-372 (1981).

[27] P.L. RoE. Upwind differencing schemes for hyperbolic conservation laws with source terms, In: Nonlinear Hyperbolic Problems. Proc. Adv. Res. Workshop, St. Étienne, 1986. Lect. Notes Math. Springer, Berlin 1270 41-51 (1987).

[28] E.F. TORO. Riemann Solvers and Numerical Methods for Fluid Dynamics, SpringerVerlag, Berlin (1997).

[29] E.F. Toro. Shock Capturing Methods for Free Surface Shallow Flows, Wiley and Sons, United Kingdom (2001).

[30] M.E. VAZQueZ-CEndon. Improved treatment of source terms in upwind schemes for the shallow water equations in channels with irregular geometry, Journal of Computational Physics 148 497-526 (1999). 